\title{
Transcription Factors Involved in the Development and Prognosis of Cardiac Remodeling
}

\author{
Jia-Hui Hong and Hai-Gang Zhang * \\ Department of Pharmacology, College of Pharmacy, Army Medical University (Third Military Medical University), Chongqing, \\ China
}

To compensate increasing workload, heart must work harder with structural changes, indicated by increasing size and changing shape, causing cardiac remodeling. However, pathological and unlimited compensated cardiac remodeling will ultimately lead to decompensation and heart failure. In the past decade, numerous studies have explored many signaling pathways involved in cardiac remodeling, but the complete mechanism of cardiac remodeling is still unrecognized, which hinders effective treatment

OPEN ACCESS

Edited by: Mahmoud El-Mas, Alexandria University, Egypt

Reviewed by:

David Zebrowski,

The Chinese University of Hong Kong, Hong Kong SAR, China

David Dolivo,

Northwestern University,

United States

Jeffrey Wigle,

University of Manitoba, Canada

*Correspondence:

Hai-Gang Zhang

hg2ster@gmail.com

hgzhang@tmmu.edu.cn

Specialty section:

This article was submitted to Cardiovascular and Smooth Muscle Pharmacology, a section of the journal Frontiers in Pharmacology

Received: 03 December 2021 Accepted: 14 January 2022

Published: 02 February 2022

Citation:

Hong $\mathrm{J}-\mathrm{H}$ and

Zhang H-G (2022) Transcription Factors Involved in the Development and Prognosis of Cardiac Remodeling.

Front. Pharmacol. 13:828549.

doi: 10.3389/fphar.2022.828549 and drug development. As gene transcriptional regulators, transcription factors control multiple cellular activities and play a critical role in cardiac remodeling. This review summarizes the regulation of fetal gene reprogramming, energy metabolism, apoptosis, autophagy in cardiomyocytes and myofibroblast activation of cardiac fibroblasts by transcription factors, with an emphasis on their potential roles in the development and prognosis of cardiac remodeling.

Keywords: transcription factors, cardiac hypertrophy, cardiac remodeling, cardiac fibrosis, fetal gene, autophagy, apoptosis

\section{DUAL ROLES OF CARDIAC REMODELING}

The basic function of the heart is pumping blood to peripheral organs to maintain their different physiological functions. The heart must work harder in the presence of an increasing workload than under normal conditions. In response to harder work, some structural changes happen in heart, indicated by size increasing and shape changing. This process is called cardiac remodeling. During cardiac remodeling, cardiomyocytes are centric cells, while other cells, like fibroblasts, also participate in this process. Both types of cardiac remodeling, i.e., physiological and pathological, are adaptive changes that maintain cardiac output under cardiac stress, but they differ in terms of the cellular mechanism and consequences (Wu et al., 2017). Physiological remodeling is characterized by a preserved or increased contractile ability induced by reversible cardiomyocyte growth in both width and length, and slight increases in heart mass without fibrosis and cell death. This type of harmless and adaptive cardiac hypertrophy often happens in growth, practice, and pregnancy (Schuttler et al., 2019). Conversely, pathological remodeling is characterized by a dilated ventricle with a thinner wall induced by increasing the cardiomyocyte length. Consequently, it progresses to systolic dysfunction, cardiac fibrosis, and heart failure. Multiple risk factors for pathological remodeling have been identified, such as hypertension, aortic stenosis, myocardial infarction, genetic cardiomyopathy and several systemic diseases (Wu et al., 2017).

As mentioned above, cardiomyocyte play a critical role in cardiac remodeling. In pathological condition, increasing cardiomyocytes cell sizes lead to heart size increase, which is called cardiac hypertrophy. Pathological cardiac hypertrophy often indicates a poor prognosis. Initially, the 


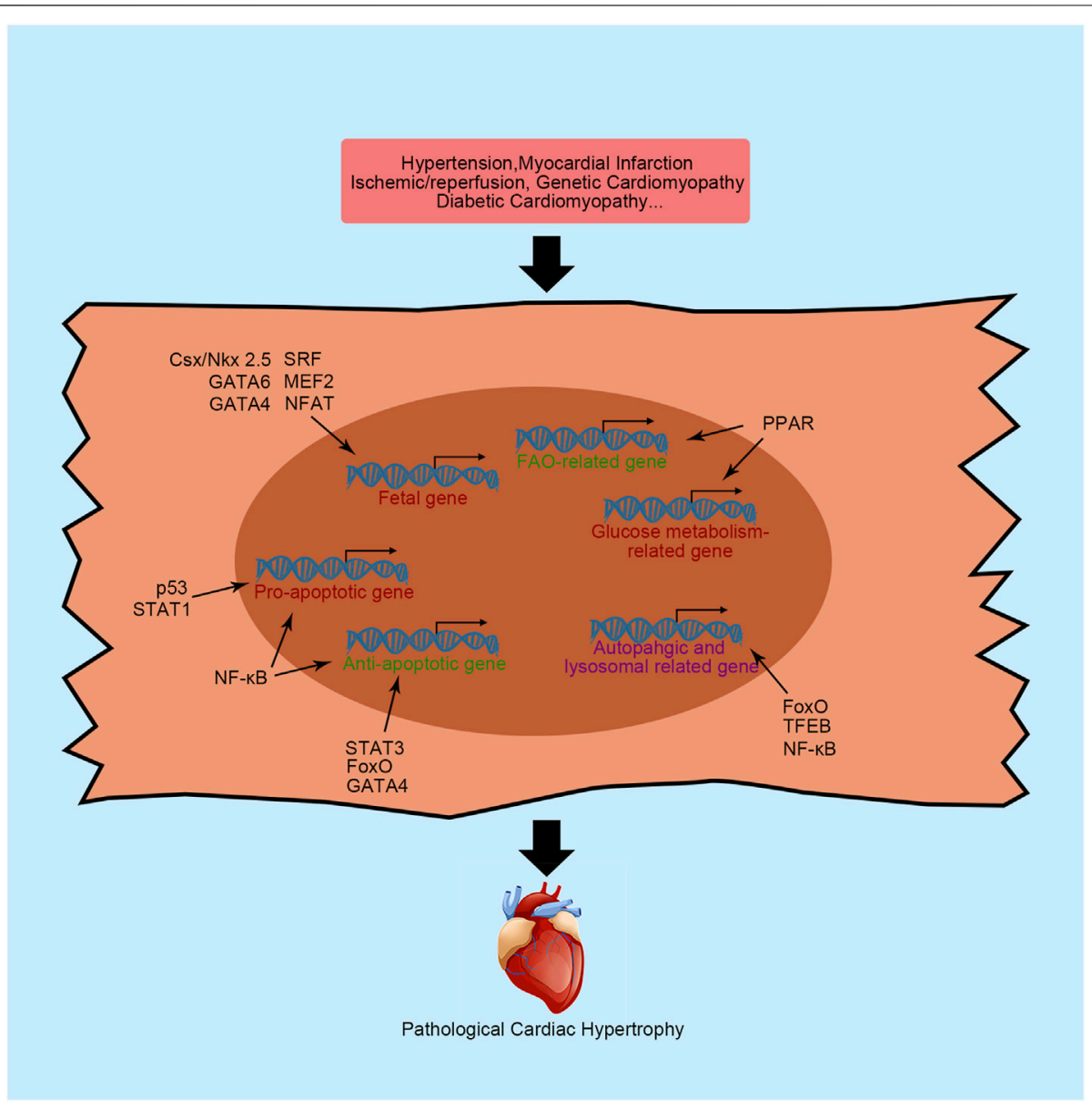

FIGURE 1 | Primary transcription factors and their functions in cardiac hypertrophy. NFAT, nuclear factor of activated T-cells; MEF2, myocyte enhancer factor 2; Csx/NKX2-5, NK-2 transcription factor related, locus 5; SRF, serum response factor; $\beta$-MHC, cardiac muscle $\beta$-isoform; ANP, natriuretic peptide A; BNP, natriuretic peptide B; PPAR, peroxisome proliferator activator receptor; FAO, fatty acid oxidation; NF- $\mathrm{B}$, nuclear factor $\kappa \mathrm{B}$; STAT, signal transducers and activators of transcription; FoxO, forkhead domain transcription factor O; TFEB, transcription factor EB; SMAD, small mothers against decapentaplegic; MRTF, myocardinrelated transcription factor.

hypertrophic response increases myocardial contractility, reduces wall stress and maintains cardiac output through compensatory mechanisms. However, the continued presence and evolving hypertrophy eventually lead to decompensation of heart function, resulting in heart failure, malignant arrhythmia, and even sudden death (Oldfield et al., 2020; Liu et al., 2021). According to an investigation by the Sarcomeric Human Cardiomyopathy Registry with 24,000 person-years of followup, patients with cardiac hypertrophy had an approximately 3fold higher mortality risk compared with similarly aged individuals in the United States general population (Virani et al., 2020). In addition, patients often suffer from a substantial economic burden and have a low quality of life. However, no effective cure is available to reverse pathological cardiac hypertrophy.

Besides cardiomyocytes, cardiac fibroblasts also participate in cardiac remodeling process with critical function. In physiological condition, cardiac fibroblast is an essential cell type in heart, which is mainly responsible for homeostasis of extracellular matrix protein (ECM). Pathologically, activated cardiac fibroblasts, also known as myofibroblasts, secret excessive ECM. Due to the limited repairment capacity of heart, excessive ECM accelerates the progression to heart failure (Kong et al., 2014). Like pathological cardiac hypertrophy, cardiac fibrosis also is an irreversible process with limited clinical treatment assays.

\section{FUNCTION OF TRANSCRIPTION FACTORS IN CARDIAC REMODELING}

Many studies have indicated that several critical signaling pathways are responsible for the remodeling process, such as the calcineurin/nuclear factor of activated T-cells (NFAT) pathway, Janus kinase/signal transducer and activator of transcription (STAT) pathway, and mitogen-activated protein kinase (MAPK) pathway (Shimizu and Minamino, 2016). Activation of these pathways requires extracellular 


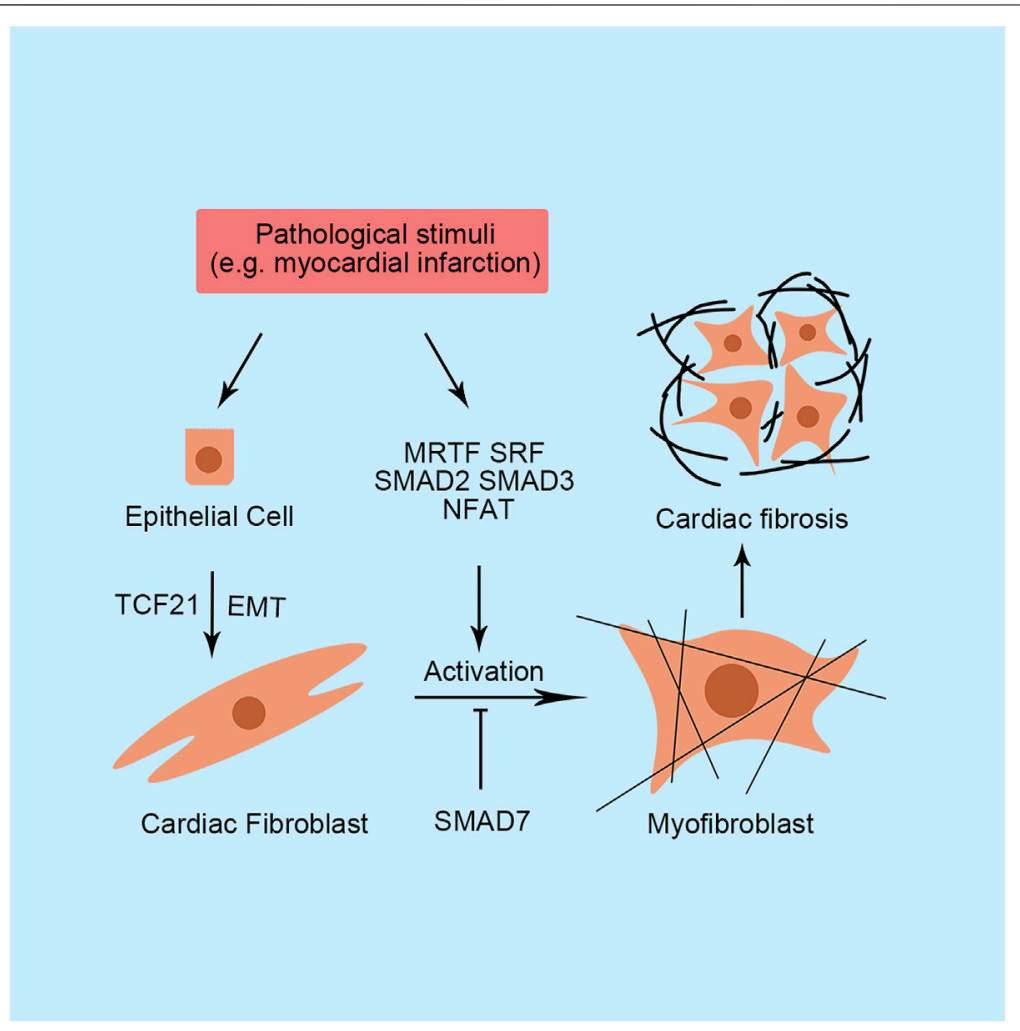

FIGURE 2 Primary transcription factors and their functions in cardiac fibrosis. SMAD, small mothers against decapentaplegic; SRF, serum response factor; MRTF, myocardin-related transcription factor; NFAT, nuclear factor of activated T-cells; TCF21, transcription factor 21; EMT, epithelial-to-mesenchymal transition.

TABLE 1 | Transcription factors and their primary target genes in the regulation of cardiac remodeling.

\begin{tabular}{|c|c|c|c|}
\hline Function & $\begin{array}{l}\text { Transcription } \\
\text { factor }\end{array}$ & Target genes & References \\
\hline \multirow[t]{5}{*}{ Expression of fetal genes } & NFAT & Nppb, Adss1, Ppp3cb, miR-25, miR-23a, and miR-199b & Molkentin et al. (1998), Luo et al. (2021) \\
\hline & GATA & $\begin{array}{l}\text { Nppa, Nppb, Myh7, Myh6, Tnnc1, Tnni1, Ncx, Adora1, Chrm2, Mlc1 and } \\
\text { Mlc3 }\end{array}$ & Zeisberg et al. (2005) \\
\hline & MEF2 & Nppa and Myh7 & Zuo et al. (2015) \\
\hline & CsX/NKX2-5 & Nppa, Adss1, Calr, Gja5, Ncx and Mef2c & Lints et al. (1993) \\
\hline & SRF & c-fos, Nppa, Nppb, Myh6, Myh7, and Ncx1 & Nelson et al. (2005) \\
\hline Energy metabolism & PPARa & Acox1, Cd36, Cpt1b, Acs/3, Cpt1a, Hmgcs2, Pcx, and Plin2 & Oka et al. (2015) \\
\hline \multirow[t]{6}{*}{ Apoptosis } & $N F-\kappa B$ & Bax, Fas, Faslg, Tp53, and miR-30b & Dolcet et al. (2005), Wei et al. (2014) \\
\hline & STAT1 & Fas and Faslg & Eid et al. (2018) \\
\hline & STAT3 & Bc/2/1 and Sod & Harhous et al. (2019) \\
\hline & p53 & Bax and IncRNA-Meg3 & Gogna et al., 2013; Li X et al. (2019) \\
\hline & FoxO & Bnip3, Bax, Sod, Cited2 and ARC & Sengupta et al. (2011), Lu et al. (2013) \\
\hline & GATA4 & $\mathrm{Bcl} 2$ & Tony et al. (2015) \\
\hline \multirow[t]{3}{*}{ Autophagy } & TFEB & Atg9. Vps11, and Lamp1 & Settembre and Ballabio (2011) \\
\hline & $N F-\kappa B$ & Becn1 & Zeng et al. (2013) \\
\hline & FoxO & Atg8, Atg12, Atg4b, Pik3c3, Gabarap/1 and Becn1 & Ferdous et al. (2010) \\
\hline \multirow[t]{4}{*}{ Fibrosis } & SMAD & Col1a, Acta2 and Tagln & Lighthouse and Small (2016) \\
\hline & MRTF & Col1a2 & Small et al. (2010) \\
\hline & NFAT & Col3 and Mrtfa & Herum et al. (2013) \\
\hline & SRF & Acta2 and Col1 & Bretherton et al. (2020) \\
\hline
\end{tabular}

NFAT, nuclear factor of activated T-cells; MEF2, myocyte enhancer factor 2; CsX/NKX2-5, NK-2 transcription factor related, locus 5; SRF, serum response factor; PPAR $\alpha$, peroxisome proliferator activator receptor $\alpha$; NF- $k B$, nuclear factor $\kappa B$; STAT, signal transducer and activator of transcription; FoxO, forkhead domain transcription factor $O$; ARC, apoptosis repressor with caspase recruitment domain; TFEB, transcription factor EB; SMAD, small mothers against decapentaplegic; MRTF, myocardin-related transcription factor. 
hypertrophic stimulation, such as pressure overload and energy starvation, and results in changes in the transcriptional program and the alternative synthesis of nucleic acids and proteins. Therefore, transcription factors play a critical role in the regulation of cardiac hypertrophy because they directly bind to DNA sequences within promoters or enhancers to control transcription. A cardiac transcription factor is defined as a protein that regulates transcription in the heart to ensure that genes are expressed in the right cell at the right time in the right amount. Currently, numerous studies have established that cardiac transcription factors influence the cardiac remodeling process by regulating hypertrophy-related gene expression or fibroblasts activation-related gene expression (Figures 1, 2, and Table 1). In this review, we outline some important transcription factors regulating fetal genes, energy metabolism, apoptosis, and autophagy in cardiomyocytes and activation in cardiac fibroblasts, which may provide a new perspective for further exploration.

\section{PROFILE AND MECHANISM OF TRANSCRIPTION FACTORS IN THE PROCESS OF CARDIAC REMODELING}

\subsection{Transcriptional Regulation of the Fetal Gene Program}

Pathological cardiac hypertrophy often manifests as the reactivation of a "fetal gene program" characterized by the upregulated expression of fetal genes, including $\beta$-isoform of cardiac myosin heavy chain $(\beta-\mathrm{MHC})$, atrial natriuretic peptide (ANP), and brain natriuretic peptide (BNP). Although the activation of these genes seems to be salutary at the early stage of cardiac hypertrophy, the aberrant expression of fetal genes results in abnormal function of cardiac contractility, calcium hemostasis, cell growth and finally heart dysfunction. Therefore, the amplified fetal genes expression helps doctors to assess cardiac function (Ingles et al., 2019). For instances, NT-proBNP has been identified as an accessory biomarker for assessing cardiac function. Interestingly, when cardiac hypertrophy is ameliorated, fetal gene expression will be significantly reduced. Here, we will introduce some transcription factors governing the expression of fetal genes.

\subsubsection{Nuclear Factor of Activated T-Cells}

NFAT is an important protein involved in an intercellular signaling pathway that regulates the expression of fetal genes. The calcineurin-regulated NFAT family consists of five family members, NFAT1-5. All of them are expressed in the heart. After dephosphorylation by calcineurin, NFAT translocates into the nucleus, binds to the specific sequence and recruits transcriptional elements. Subsequently, fetal genes are reexpressed and activated. The function of NFAT in embryos is well understood. The lack of NFAT in fetal development induces a loss of contractile protein and a thin atrial wall in mice (Schubert et al., 2003). However, when this fetal gene program governor is reactivated in the adult mammalian heart, a hypertrophic response occurs concomitantly. In human, the increasing dephosphorylation of NFAT3 is related with dilated cardiomyopathy (DCM) (Diedrichs et al., 2004). Suppression of NFAT activity by nuclear localized protein 1 effectively attenuates isoprenaline-induced cardiac hypertrophy in mice, as indicated by reduced cardiac fibrosis, improved cardiac contractility function and preservation of left ventricular geometry, which suggested the harmful role of NFAT in the adult heart (Zhang et al., 2020). Nppb is one of the genes regulated by NFAT in cardiomyocytes. Using neonatal rats ventricular myocytes (NRVMs), Molkentin et al. (1998) found that the $N p p b$ promoter was activated 100-fold more in the presence of NFAT with the coactivator GATA-4. Furthermore, overexpression of calcineurin leading to constitutive activation of NFAT dramatically increases the expression of BNP transcripts and induces serious cardiac hypertrophy and fibrosis in mice. In addition to $N p p b$, regulator of calcineurin 1.4 is validated as a direct target of NFAT in NRVMs (Luo et al., 2021). Recently, the role of NFAT in epigenetics was noted. NFAT controls many microRNAs transcription, such as miR-23a and miR-223 (Luo et al., 2021). These microRNAs controlled by NFAT are positively correlated with pathological cardiac hypertrophy (Chen et al., 2017).

\subsubsection{GATA Transcription Factors}

GATA is another transcription factor superfamily that exerts critical regulatory functions in the heart. Six known members of the GATA family have been detected in vertebrates. GATA1/2/3 are preferentially expressed in hematopoietic cells, while GATA4/5/6 proteins are expressed in various tissues, including the liver, lung, gut, gonad and heart. Among the GATA superfamily members, GATA4 is the most extensively explored transcription factor in the heart (Whitcomb et al., 2020). Similar to NFAT, inactivation of GATA4 in mouse embryos leads to death by E8.5 due to abnormal heart development (Zeisberg et al., 2005). Combined with other transcription factors, GATA4 directly regulates the expression of numerous genes in the heart, including Nppa, Nppb, Myh7, Myh6, Tnnc1, Tnni1, Ncx, Adora1, Chrm2, Mlc1, and Mlc3 (Zeisberg et al., 2005). Upregulated GATA4 expression has been found in human DCM heart (Diedrichs et al., 2004). In adult hearts, transgenic mice with cardiomyocytes-specific overexpression of GATA4 by 2.5 -fold displayed cardiac hypertrophy (Liang et al., 2001). Expression of a dominant negative GATA4 by a GATA4 engrailed repressor fusion-encoding adenovirus ameliorates phenylephrine-induced cardiac hypertrophy and remodeling in NRVMs (Liang et al., 2001). The mechanism was further studied by mutating serine 105 to alanine in GATA4 (GATA4S105A), which is a known GATA4 phosphorylation site. GATA4-S105A mice did not exhibit cardiac hypertrophy induced by 2 weeks of $\mathrm{PE}$ infusion with decreased expression of $N p p a, N p p b$ and $M y h 7$, signifying that extracellular signal-regulated kinase $1 / 2$ and p38 MAPK dependent phosphorylation of GATA4 were required for fetal gene reactivity in hypertrophic hearts (van Berlo et al., 2011). In addition, acetylation was necessary for GATA4 to exert hypertrophic effects. Deacetylation of GATA4 by Sirtuin 
7 significantly ameliorates transverse aortic constriction (TAC)-induced cardiac hypertrophy in mice (Yamamura et al., 2020).

Over 30 mutations in GATA6 gene are associated with congenital heart disease, indicating the pivotal regulating function of GATA6 in heart (Whitcomb et al., 2020). Both gain-of-function and loss-of-function studies have established a critical role for GATA6 in cardiomyocytes. Forced expression of GATA6 in mouse cardiomyocytes results in an increased cell surface area, while cardiomyocytes specific GATA6 deletion leads to decreased fetal gene expression and reduced cardiac hypertrophy caused by pressure overload stimulation in mice (van Berlo et al., 2010). The functions of GATA4 and GATA6 partially overlap. Neonatal GATA4 and GATA6 inactivation in mouse hearts causes more rapid and severe heart failure than single knockout (Liang et al., 2001). However, GATA4 and GATA6 do not completely compensate for each other. Hybridized mice with knockout of GATA4 or GATA6 and overexpression of the other protein displayed partially reversed cardiac hypertrophy without full heart function recovery (van Berlo et al., 2013).

\subsubsection{Myocyte Enhancer Factor 2}

The myocyte enhancer factor 2 (MEF2) superfamily consists of four members, namely, MEF2A-D. During mouse embryonic development, $\mathrm{MEF} 2 \mathrm{~B}$ and $\mathrm{MEF} 2 \mathrm{C}$ are first expressed in the cardiac mesoderm at E7.5, followed by MEF2A and MEF2D expression a day later (Desjardins and Naya, 2016). The heterozygous mutation of $\mathrm{MEF} 2 \mathrm{C}$ in human increased susceptibility to DCM (Yuan et al., 2018). In addition, upregulation of MEF2 is related to cardiac hypertrophy in mice adult hearts. Several fetal genes have been identified as direct target genes of MEF2, including Nppa and Myh7 (Zuo et al., 2015). Knockdown of MEF2C or MEF2D in mice is sufficient to blunt hypertrophic heart growth in response to pressure overload, while the overexpression of MEF2A, MEF2C and MEF2D causes ventricular dilation and cardiac dysfunction (Clapham et al., 2019). The robust activation of MEF2 in cardiac hypertrophy is associated with histone deacetylases (HDACs), which promote chromatin condensation and subsequent transcriptional repression (Zhang et al., 2002). Cardiac hypertrophic stimulation is sufficient to induce HDAC phosphorylation, which promotes the nuclear export of HDAC and transcriptional activity of MEF2. Mutation of the phosphorylation site of HDAC9 renders mouse cardiomyocytes intolerant to PE-induced cardiac hypertrophy and upregulates ANP and $\beta-\mathrm{MHC}$ expression. However, HDAC9-null mice display an increased sensitivity to hypertrophic stimuli, such as pressure overload, indicating that unphosphorylated HDAC continually suppresses MEF2 transcriptional activity (Zhang et al., 2002).

\subsubsection{NK-2 Transcription Factor Related, Locus 5}

The homeodomain-containing transcription factor Csx/NKX2-5 belongs to the NK homeobox gene family, and functions as a transcriptional activator with an indispensable regulatory role in embryo heart development and adult heart function. The name of Csx/NKX2-5 consists of two parts. "Csx" is the acronym for cardiac-specific homeobox, indicating the critical biological function in myocardial cells, while NKX2-5 represents the fifth vertebrate gene identified in the NK-2 homeobox gene family. The critical role of Csx/NKX2-5 in developmental biology has been extensively established (Chen and Fishman, 1996). The direct downstream target genes of Csx/NKX2-5 have also been extensively explored, such as Nppa, Adss1, calreticulin, connexin40, Ncx1, and MEF2C (Lints et al., 1993). The regulatory mechanism of $\mathrm{Csx} / \mathrm{NKX} 2-5$ often synergizes with other transcription factors, such as GATA4. Using COS-9 cell lines, Shiojima et al. (1999) found that expression of both Csx/ NKX2-5 and GATA4 results in the much higher transcription of Nppa compared with Csx/NKX2-5 expression alone, indicating that Csx/NKX2-5 synergized with GATA4 to initiate Nppa transcription. In humans, mutations in Csx/NKX2-5 are strongly associated with congenital heart diseases (Wang et al., 2011). Taken together, this evidence supports a critical role for Csx/NKX2-5 in the fetal gene program and cardiac hypertrophy.

\subsubsection{Serum Response Factor}

As previously mentioned, SRF also regulates fetal gene expression with the help of some cofactors, such as GATA4 and Csx/Nkx2-5. SRF has been discovered in regulating several gene expression including cell growth, differentiation, migration, etc., (Niu et al., 2005). Cardiac-specific knockout of SRF in embryos leads to cardiac insufficiency and embryonic lethality (Niu et al., 2005). In the postnatal heart, the presence of SRF is required for the expression of $c$-fos, Nppa, Nppb, Myh6, Myh7, and Ncx1 (Nelson et al., 2005). However, the function of SRF in the regulation of cardiac hypertrophy is self-contradictory. Both gain and loss of SRF function induce cardiac hypertrophy (Liu et al., 2015). Using heart specific tamoxifen-induced Cre recombinase, cardiomyocytes specific disruption of SRF in mice causes impaired left ventricular function with decreased contractility. Besides, all mutant mice died from serious heart failure 10 weeks after treatment, indicating the harmful role of SRF in cardiomyocytes (Parlakian et al., 2005). However, transgenic mice with cardiomyocytes-specific overexpressing human SRF also leads to serious cardiac hypertrophy (Zhang et al., 2001). In addition, SRF expression levels are decreased in subjects with PE-induced cardiac hypertrophy but increased in aortic banding-induced cardiac hypertrophy (Nelson et al., 2005; Liu et al., 2015). Recently, Li et al. (2020) reported that phosphorylation of SRF significantly decreased in dilated cardiomyopathy patients. Besides, the activated caspase 3 cleaves SRF and produce a domain-negative transcription factor, suggesting that increasing SRF expression may benefit the DCM patients (Chang et al., 2003).

\subsection{Regulation of Energy Metabolism and Peroxisome Proliferator Activator Receptors}

The heart is a typical organ with a high energy consumption. Two primary sources of continuous and extensive energy production in cardiomyocytes have been shown to sustain the heartbeat: mitochondrial oxidative phosphorylation and glycolysis. The 
former accounts for approximately 95\% of myocardial ATP consumption, while the latter accounts for approximately $5 \%$. After exposure to different stresses, the heart utilizes diverse ranges of substrates to produce energy. In normal hearts, approximately $40-60 \%$ ATP is produced using fatty acids as fuel (Karwi et al., 2018). In addition, lactate, glucose, ketones, and amino acids are also common substrates for energy production in the heart. Generally, a dramatic alteration in energy metabolism occurs in hypertrophic and failing hearts (Karwi et al., 2018). This disordered energy metabolism is caused by several factors, including impaired mitochondrial oxidative metabolism, alterations in energy substrate preference by the heart, and a decrease in cardiac efficiency. A decrease in ATP production is a characteristic of the failing heart and is primarily caused by impaired mitochondrial oxidative capacity. Compared with a healthy heart, approximately $30 \%$ less ATP production is detected in the end-stage failing heart (Karwi et al., 2018). Furthermore, changes in the energy pattern are associated with alterations in the transcriptional program. Indeed, fatty acid oxidation (FAO)-related genes are downregulated in hypertrophic and failing hearts of hypertensive rats compared to controls (Karwi et al., 2018).

Alterations in cardiac energy production contribute to the severity of heart failure. The peroxisome proliferator activator receptor (PPAR) superfamily is an important and well-known protein superfamily affecting heart energy metabolism by regulating transcription. All three isoforms of PPAR, PPARa, $\operatorname{PPAR} \beta / \delta$, and PPAR $\gamma$, are expressed in the heart. PPAR activation is characterized by the formation of transcriptional complexes with ligands, retinoic acid-x-receptors and coactivators. Subsequently, the active complex directly binds to the specific DNA binding element to recruit related enzymes, remodel chromatin, modify histones and initiate transcription (Ajith and Jayakumar, 2016).

\subsubsection{PPAR $a$}

The function of PPARa in the heart has been studied extensively using gain-of-function and loss-of-function methods. The rate of fatty acid metabolism decreases along with an increase in the glucose consumption rate in the hearts of PPARa ${ }^{-/-}$mice (Oka et al., 2015). In addition, PPARa-deficient mice display reduced cardiac function and impaired compensation for an increased workload (Khuchua et al., 2018). This phenotype was partially caused by lipid metabolite accumulation and energy starvation. In PPARa-overexpressing mice, several PPAR target genes, such as Acox1, Cd36, Cpt1a, Cpt1b, Acsl3, Hmgcs2, Pcx, and Plin2, are upregulated (Oka et al., 2015). Fatty acid metabolism-related enzymes are upregulated along with decreased expression of glucose uptake- and oxidation-related proteins in mice with cardiomyocytes-restricted overexpression of PPARa (MHCPPARa) (Finck et al., 2002). Using isolated working hearts and micro-positron emission tomography analyses, Finck et al. (2002) established that the myocardium predominantly uses fatty acids as energy resources instead of glucose uptake and metabolism when PPARa is overexpressed in cardiomyocytes. Taken together, these findings suggested that PPAR $\alpha$ activation in cardiomyocytes increases fatty acid uptake, storage, and utilization by controlling the expression of some critical genes in the FAO pathway. However, researchers have been unable to conclude that downregulated PPAR $\alpha$ is associated with cardiomyopathy. First, different levels of PPAR $\alpha$ expression are observed in individuals with $\mathrm{HF}$ characterized by FAO deficiency, suggesting that downregulated PPAR $\alpha$ is not a common feature of heart failure (Khuchua et al., 2018). Second, both cardiomyocytes-specific knockout and overexpression of PPARa induce cardiac hypertrophy in mouse hearts, which indicates the controversial role of PPARa in the development of cardiac hypertrophy (Finck et al., 2002; Khuchua et al., 2018). In addition, the activation of PPARa in different animal models leads to different consequences. Administration of WY14643, a specific PPARa ligand, in the early stage of severe myocardial damage induced by diabetes leads to a higher mortality rate than the vehicle group (Chen et al., 2010). However, PPARa activation in the early stage of TACinduced mouse heart failure preserves myocardial function (Kaimoto et al., 2017). Last, the effect of PPARa on regulating Glut4, a glucose transporter promoting glucose uptake, remains controversial. In MHC-PPARa mice, the upregulated PPARa exerts an inhibitory effect on Glut4, while increased PPAR $a$ activation increases Glut4 expression synergistically with the PPAR $\alpha$ coactivator fenofibrate after administration to mice, signifying the complicated role of PPARa in regulating cardiac glucose metabolism (Burkart et al., 2007; Yao et al., 2012). Taken together, all the studies conducted to date have revealed the complex regulatory mechanism of PPARa in the diseased heart.

\subsubsection{PPAR $\beta / \delta$}

Both PPAR $\beta / \delta$ and PPAR $\gamma$ play similar roles to PPAR $\alpha$ in cardiomyocytes. All of these proteins govern FAO-related gene expression in cardiomyocytes, including fatty acid uptake, mitochondrial fatty acid $\beta$-oxidation, malonyl-CoA metabolism, and peroxisomal oxidation (Burkart et al., 2007). The specific deletion of PPAR $\beta / \delta$ in mouse cardiomyocytes leads to heart dysfunction and reduced fatty acid metabolism, signifying the regulatory effect of $P P A R \beta / \delta$ on FAO in the heart (Chen et al., 2013). Interestingly, PPAR $\beta / \delta$ activation increases FAO-related and glucose oxidation-related gene expression (Wang et al., 2010). As a result of these changes, cardiomyocytes-restricted PPAR $\beta / \delta$ overexpressing mice fed a high-fat diet do not have cardiac myopathy induced by lipid accumulation compared with MHC-PPARa diabetic mice (Burkart et al., 2007). Notably, the level of the endogenous antioxidant $\mathrm{Cu} / \mathrm{Zn}$ superoxide dismutase is also increased after the constitutive activation of PPAR $\beta / \delta$, indicating that PPAR $\beta / \delta$ activation protects the heart from oxidative stress (Barlaka et al., 2015). All this evidence suggests a protective role for PPAR $\beta / \delta$ in the heart. Indeed, the PPAR $\beta / \delta$ activation rescues in heart dysfunction in mice after ischemic/reperfusion (I/R) injury compared with wild-type mice, and $\operatorname{PPAR} \beta / \delta$ activation protects neonatal rat cardiomyocytes from hypertrophy. Consistent with the in vitro findings, PPAR $\beta / \delta$ overexpression in the cardiomyocytes improves cardiac function and reduces fibrosis and energy metabolism in mice with pressure overloadinduced cardiac hypertrophy (Wang et al., 2010). Therefore, the 
administration of $\mathrm{PPAR} \beta / \delta$ ligands might be a potential therapeutic method for cardiomyopathy in the future.

\subsubsection{PPAR $\gamma$}

Many researchers have explored the function of PPAR $\gamma$ in cardiomyocytes. Compared with adipose-enriched tissue, PPAR $\gamma$ is expressed at a lower level in the heart. PPAR $\gamma$ agonists, such as thiazolidinediones, are widely used in the clinic as insulin sensitizers to treat diabetes and exhibit good efficacy in preventing cardiac vascular events. However, emerging evidence has shown that PPAR $\gamma$ agonists may not adequately protect the heart (Wright et al., 2014). In the human heart, upregulated PPAR $\gamma$ expression has been detected in the left ventricle and coronary arteries of patients with cardiomyopathy and coronary heart disease compared with normal people (Mehrabi et al., 2003). Paradoxically, Liu et al. (2016) revealed that quercetin protected the heart from I/R-induced damage via PPAR $\gamma$ activation in $\mathrm{H} 9 \mathrm{C} 2$ cells, indicating the protective role of PPAR $\gamma$ in the heart. The conflicting results may be attributed to the off-target effects of PPAR $\gamma$ agonists. Cardiomyocytes-specific knockout of PPAR $\gamma$ leads to mild cardiac hypertrophy with preserved heart function (Duan et al., 2005). After treatment with rosiglitazone, a PPAR $\gamma$ agonist, cardiomyocytes-specific PPAR $\gamma$-null mice display serious cardiac hypertrophy and cardiac dysfunction, indicating the indirect hypertrophic effect of PPAR $\gamma$ agonists (Duan et al., 2005). Using cardiac specific PPAR $\gamma$ overexpressing mice, Son et al. (2007) found that cardiomyocytes-specific overexpression of PPAR $\gamma$ caused all mice to die at 5 months of age from severe cardiac hypertrophy due to high fatty acid uptake and metabolism. Additionally, the expression of glucose uptake-related genes did not decrease. Interestingly, when cardiomyocytes-specific PPAR $\gamma$-overexpressing mice were crossed with PPARa-deficient mice, cardiac hypertrophy was significantly ameliorated, along with upregulated FAO and decreased apoptosis, reactive oxygen species (ROS) levels, and endoplasmic reticulum stress (Son et al., 2010). These complex and incompatible findings regarding the function of PPAR $\gamma$ suggest that suitable expression of PPAR $\gamma$ benefits the heart. Researchers have not clearly determined whether excessively high or low PPAR $\gamma$ expression in cardiomyocytes induces cardiac hypertrophy and dysfunction.

\subsection{Regulation of Apoptosis}

Apoptosis refers to a process of programmed cell death. Physiologically, apoptosis plays a critical role in maintaining homeostasis by destroying unnecessary cells. However, excess apoptosis will result in pathological conditions and finally lead to organ failure. Researchers concluded that reduced cardiomyocytes apoptosis protects the heart from cardiac dysfunction and remodeling during pathological conditions, such as myocardial infarction (MI) and I/R injury (Teringova and Tousek, 2017). The expression of several apoptosis-related genes is upregulated in the cardiomyocytes under pathological conditions, while the expression of antiapoptotic genes is suppressed. These processes require the involvement of transcription factors. Here, we will introduce some critical transcription factors involved in regulating cardiomyocytes apoptosis.

\subsubsection{Nuclear Factor- $\kappa B$}

Nuclear factor $-\kappa B(N F-\kappa B)$ is one of the core transcription factors in the apoptosis pathway and was first discovered to regulate immunoglobulin $\kappa \mathrm{B}$ expression in $\mathrm{B}$ lymphocytes. The mammalian NF- $\kappa \mathrm{B}$ superfamily contains five members, RelA (p65), RelB, c-Rel, p50 and p52, which are characterized by conserved Rel homology domain at the N-terminus. Further exploration suggested that NF- $\kappa \mathrm{B}$ participates in various of cellular signaling pathways in multiple cell types (Zhang et al., 2017). Among all of these signaling pathways, inhibitor of nuclear factor $\kappa \mathrm{B}(\mathrm{I} \kappa \mathrm{B})$ is a key regulator that directly controls $N F-\kappa B$ activation. Classically, NF- $\kappa B$ activation depends on the $I \kappa B$ kinase (IKK)-mediated phosphorylation of $I \kappa B$. Subsequently, $\mathrm{NF}-\kappa \mathrm{B}$ translocates into the nucleus and binds to specific DNA sequences. NF- $\kappa \mathrm{B}$ exerts a critical effect on regulating cardiomyocyte apoptosis, especially in response to oxidative stress following myocardial infarction (Imam et al., 2018). In human, Czibik et al. (2008) reported that NF- $\kappa \mathrm{B}$ nuclear translocation was reduced at reperfusion in stable angina patients after ischemic precondition. However, researchers have not conclusively determined whether it exerts cardioprotective effects or deleterious effects. Indeed, $N F-\kappa B$ activation induces the expression of both proapoptotic proteins (e.g., Bax, Fas, FasL and p53) and antiapoptotic proteins (Dolcet et al., 2005). The cardiomyocytes-specific deletion of NF- $\kappa \mathrm{B}$ essential modulator/IKK $\gamma$ in mice causes spontaneous ventricular dilation with increased oxidative stress and apoptosis levels (Kratsios et al., 2010). Furthermore, the direct deletion of p50 in mice exacerbates cardiac ventricular dilation, inflammation and fibrosis (Timmers et al., 2009). However, in contrast, other studies reported that p50 deficiency in mice significantly improves survival and cardiac hypertrophy following infarction without decreasing inflammatory cytokine expression (Frantz et al., 2006). Using an I $\mathrm{KBa}$ mutation to specifically mask the nuclear localization sequence of p65, Hamid et al. found that reduced activity of p65 in mouse cardiomyocytes alleviates pathological cardiac hypertrophy by increasing stress-induced apoptosis (Hamid et al., 2011). In addition, they recognized that p65 is mainly responsible for $\mathrm{NF}-\mathrm{\kappa B}$ activity to initiate cardiac remodelingrelated gene transcription (Hamid et al., 2011). Combined with the p50 deficiency-induced upregulation of p65 in fibroblasts from embryonic mice stimulated with lipopolysaccharide, controversial experimental results for p50 showed compensatory p65 activation (Timmers et al., 2009; Hamid et al., 2011). Currently, accumulating evidence has proven that NF- $\kappa \mathrm{B}$ downregulation benefits the heart by reducing inflammation in both H9C2 cells, NRVMs and human (Mangali et al., 2019; Luo et al., 2020; Fan et al., 2021). Notably, NF- $\kappa \mathrm{B}$ plays a critical role in epigenetics. By positively controlling miR-30b, NF- $\kappa \mathrm{B}$ indirectly inhibits the expression of the antiapoptotic protein $\mathrm{Bcl}-2$, resulting in increased NRVMs death upon angiotensin II stimulation (Wei et al., 2014). 


\subsubsection{Signal Transducer and Activator of Transcription} Another important transcription factor family governing apoptosis is STAT. Seven members of the STAT family have been identified in mammals, i.e., STAT1, STAT2, STAT3, STAT4, STAT5a, STAT5b and STAT6. Although they share similar structures, their biological functions are different. Among these seven members, the main STATs functioning in the heart are STAT1 and STAT3. Therefore, we will discuss these two transcription factors in more detail. Many early studies established that STAT1 and STAT3 play critical roles in controlling cell fate by regulating the expression of apoptosisrelated genes. In human, the phosphorylation of both STAT1 and STAT3 are significantly increased in DCM (Ng et al., 2003). However, animal studies revealed that STAT1 and STAT3 exert completely opposite effects on controlling cardiomyocyte apoptosis, similar to their effects on cancer cells (Knight et al., 2012). The phosphorylation of STAT1, indicating activated STAT1, is increased in cardiomyocytes following I/R damage and upregulates proapoptotic proteins such as Fas and FasL (Eid et al., 2018). Moreover, STAT1 interacts with a well-known proapoptotic transcription factor, p53, to increase the transcriptional activity of p53 in apoptosis (Carroll et al., 2013). In contrast, STAT3 activation promotes the expression of some antiapoptotic genes (e.g., $\mathrm{Bcl}-\mathrm{xL}$ ) and antioxidant proteins (e.g., superoxide dismutase), which protect the heart from I/R injury (Harhous et al., 2019). Compared with wild-type mice, cardiomyocytes-specific knockout of STAT3 induces much more serious injury in the heart following I/R, namely, increased apoptosis of cardiomyocytes and mortality (Hilfiker-Kleiner et al., 2004). The cardioprotective role of STAT3 was further confirmed by analyzing transgenic mice overexpressing active STAT3. Mice overexpressing STAT3 are more resistant to I/R-induced damage than wild-type mice and exhibited smaller infarct areas (Oshima et al., 2005).

\subsection{3 p53}

As a proapoptotic transcription factor, p53 was first considered a tumor suppressor that also regulates the apoptosis process in cardiomyocytes. In rats with cardiac $\mathrm{I} / \mathrm{R}$ injury, p53 accumulates and is activated by acetylation, which mediates the transcription of Bax, a cell death effector, to initiate the apoptosis program (Gogna et al., 2013). In addition to the upregulation of Bax, the downstream targets of Bax are also upregulated in a rat $\mathrm{I} / \mathrm{R}$ model, including cytochrome C, apoptosis-inducing factor, cleaved caspase-3, caspase-9, and apoptotic peptidase activating factor 1 , indicating the critical function of $\mathrm{p} 53$ in regulating cardiomyocyte apoptosis (Men et al., 2021). In human, the upregulated p53 protein expression is associated with heart failure (Song et al., 1999). Therefore, upregulated p53 mediates decompensated pathological cardiac hypertrophy through p53-dependent apoptosis, while downregulated p53 rescues cardiac hypertrophy (Men et al., 2021). Raut et al. (2016) found that decreased p53 expression by miR-30c or miR-181a overexpressing attenuated diabetes-induced cardiac hypertrophy, indicated by decreased ANP expression and cardiomyocytes size. Moreover, some researchers recently reported that p53 modulates the function of some noncoding RNAs to regulate the execution of apoptosis. For example, using MI mice model, Li X et al. (2019) found that long noncoding RNA Meg3, an apoptosis inducer controlled by $\mathrm{p} 53$, binds to FUS, an apoptosis inducer, to promote apoptosis through the upregulation of the proapoptotic protein caspase-9 and downregulation of the antiapoptotic protein $\mathrm{Bcl}-2$. P53 has also been shown to participate in the endoplasmic reticulum (ER) stress-induced apoptosis pathway in cardiomyocytes. Cardiomyocytes-specific knockout of p53 decreases thapsigargin-induced ER stress and attenuates cardiomyocyte apoptosis (Chen et al., 2019). However, these inhibitory effects were incomplete, indicating that the other pathway may also participate in ER stress-induced mitochondrial damage.

\subsubsection{Forkhead Box Transcription Factor $O$ Transcription Factors}

The forkhead box transcription factor $\mathrm{O}$ (FoxO) family regulates diverse cellular signaling pathways in specific cells. Currently, four members of the FoxO family (FoxO1, FoxO3, FoxO4, and FoxO6) have been identified in mammalian cells. Emerging evidence has proven that the FoxO family controls the activation of apoptosis-related pathways by regulating the transcription of several genes, including Bnip3 and Bax (Sengupta et al., 2011). Using an oxidative stress NRVMs model, Sengupta et al. (2011) found that ROS-induced cardinal injury promotes the nuclear localization of both FoxO1 and FoxO3 (Sengupta et al., 2011). The activation of FoxO1 and FoxO3 subsequently initiates superoxide dismutase and CBP/P300-interacting trans-activator expression, which serve as antioxidant and antiapoptotic proteins, respectively, to protect cardiomyocytes from damage and apoptosis. Moreover, the apoptosis repressor with caspase recruitment domain, a potent anti-apoptosis protein, is a direct transcriptional target of FoxO3a ( $\mathrm{Lu}$ et al., 2013). Upregulated FoxO3a suppresses the release of $\mathrm{Ca}^{2+}$ from the sarcoplasmic reticulum induced in response to oxidative stress to inhibit $\mathrm{Ca}^{2+}$-mediated apoptosis by increasing the level of apoptosis repressor with caspase recruitment domain in cardiomyocytes (Lu et al., 2013).

\subsubsection{GATA4}

The critical role of the transcription factor GATA4 in cardiomyocyte apoptosis has been extensively investigated. Upregulated GATA4 is negatively correlated with cardiomyocyte apoptosis. Doxorubicin upregulates miR-208a to inhibit the expression of GATA4 and promote cardiomyocytes apoptosis in mice, while silencing of miR-208a reverses the increase in cardiomyocyte apoptosis by increasing Bcl-2 expression via GATA4 in mice (Tony et al., 2015). According to a subsequent study, GATA4 coordinates with cardiac ankyrin repeat protein to directly bind the GATA binding site located in the $\mathrm{Bcl}-2$ promoter and upregulate $\mathrm{Bcl}$ 2 expression to counter doxorubicin-induced cardiomyocyte apoptosis (Zhang et al., 2016). 


\subsection{Regulation of Autophagy}

Proteins and organelles are continuously synthesized throughout the cell lifespan. The proper degradation and turnover of proteins and organelles play pivotal roles in homeostasis and survival of cells and life. Autophagy is an evolutionarily conserved subcellular pathway for lysosome-mediated turnover of proteins and organelles that was first discovered in 1963. Basal autophagy is crucial for cardiomyocytes to maintain hearts homeostasis and normal function, permitting the clearance and recycling of obsolete and dysfunctional elements (Sciarretta et al., 2018). In particular, the cells of terminally differentiated organs are unable to remove their waste without autophagy because they do not replicate (Sciarretta et al., 2018). This function of autophagy was highlighted using cardiomyocytes-specific knockout of autophagy-related proteins. Cardiomyocytes-specific inactivation of autophagyrelated protein (ATG) 5, a crucial protein involved in autophagy, causes progressive heart failure through the accumulation of ubiquitylated proteins, mitochondrial dysfunction, and disorganized sarcomeres (Sciarretta et al., 2018). However, excess autophagy leads to cell death. The dual roles of autophagy suggest that the beneficial or detrimental function of autophagy in the heart depends on the type of stimulation and timing of the measurement. Basically, a suitable level of autophagy benefits the heart.

\subsubsection{Transcription Factor EB}

Transcription factor EB (TFEB) plays a crucial role in regulating autophagy and lysosome biogenesis and is characterized by a basic helix-loop-helix-leucine zipper motif. Dephosphorylated TFEB translocates to the nucleus and binds to specific DNA sequences to regulate the expression of autophagy- and lysosomerelated genes, such as ATG9, vacuolar protein sorting-associated protein 11, and lysosomal-associated membrane protein 1 (Settembre and Ballabio, 2011). TFEB nuclear translocation decreased in DCM patient, indicating the protective function of TFEB in heart (Caragnano et al., 2019). In young mice, increased TFEB translocation ameliorates lipopolysaccharideinduced oxidative damage by activating the autophagy cascade in cardiomyocytes, while TFEB does not translocate and initiate autophagy-related gene expression in aged mice treated with lipopolysaccharide ( $\mathrm{Li}$ et al., 2016). Similarly, glucolipotoxicity caused by diabetes and obesity in mice reduces TFEB expression in cardiomyocytes and leads to a disruption of lysosomal homeostasis, impaired cardiomyocyte proteostasis, and cardiac myopathy (Trivedi et al., 2016). TFEB overexpression protects the heart from cardiac proteotoxicity by increasing autophagy flux (Pan et al., 2017). After treatment with 3-methyladenine, a type III phosphatidylinositol 3-kinase inhibitor used to suppress autophagy, the protective effect of forced expression of TFEB vanished in NRVMs, indicating the protective effect of increased autophagy flux induced by the TFEB transgene (Pan et al., 2017). Recently, Trivedi et al. (2020) found that the protective function of TFEB in cardiomyocytes bypassed the autophagy process. The cardiomyocyte-specific deletion of TFEB aggravates nutrient overload-induced lipid droplet accumulation and caspase-3 activation, while TFEB overexpression in NRVMs exerts the opposite effect. In the presence of ATG7 loss-of-function, the protective effect of overexpressed TFEB persists, indicating that the effect of TFEB on reprogramming energy metabolism was more evident than its effect on regulating autophagy to protect cardiomyocytes (Trivedi et al., 2020). Targeting TFEB to maintain a suitable autophagy level in the diseased heart may be a novel idea for new drug development.

\subsubsection{Nuclear Factor $\kappa \mathrm{B}$}

In addition to the apoptotic pathway, NF- $\kappa \mathrm{B}$ also plays a pivotal role in autophagy. The well-known target of NF- $\kappa \mathrm{B}$ in autophagy is Beclin-1. In rabbit I/R-injured hearts, Beclin-1 upregulation, which is induced by active p62, aggravates cardiac dysfunction, especially in the cardiac area at risk (Zeng et al., 2013). Overexpression of $\mathrm{I} \kappa \mathrm{B}$, an inhibitor of the NF- $\mathrm{B}$ signaling pathway, reduces p65 nuclear translocation and is associated with decreased Beclin-1 expression and a reduced LC3-II/LC3-I ratio in mice exposed to $\mathrm{H}_{2} \mathrm{O}_{2}$, suggesting that the reduced autophagy level protects the heart from $\mathrm{H}_{2} \mathrm{O}_{2}$-induced oxidative stress (Han et al., 2020). A similar outcome was confirmed in the diabetic mice. Increased autophagy activation damaged the hearts of diabetic mice, while a p65 siRNA significantly ameliorated this injury (Wang et al., 2017).

\subsubsection{Forkhead Box Transcription Factor $O$}

Many studies have confirmed that FoxO induces the expression of multiple autophagy-related proteins, including ATG8, ATG12, ATG4B, phosphatidylinositol 3-kinase catalytic subunit type 3, GABA type A receptor associated protein like 1 and Beclin-1 (Ferdous et al., 2010). FoxO overexpression in NRVMs attenuates agonist-induced cardiac hypertrophy and increases autophagy levels. In energy-deprived cardiomyocytes, activated FoxO1 promotes autophagy-related gene expression and stimulates autophagy flux (Hariharan et al., 2010). Nonetheless, the beneficial or detrimental role of FoxO in cardiomyocytes is difficult to determine. In human, increasing FoxO3a expression was noted in heart from heart failure patients compared with control group (Galasso et al., 2010). In mice ischemic hearts, poly (ADP-ribose) polymerase-1 promotes FoxO3a activation and translocation (Wang et al., 2018). Subsequently, the activation of FoxO3a increases autophagyrelated gene expression, which further impairs mitochondrial function, increases cell death, and aggravates pathological cardiac remodeling (Wang et al., 2018).

\subsection{Regulation of Fibrosis}

In addition to cardiomyocytes, fibroblasts are an important component of the heart and contribute to $10-30 \%$ of the total cardiac cell population. Fibroblasts are mainly responsible for providing basic structural support by ECM into the myocardium. Therefore, fibroblasts play a central role in tissue repair after injury. In damaged heart, cardiac fibroblasts express excessive cytokines and growth factor, leading to excessive proliferation of cardiac fibroblasts, myofibroblasts activation, and excessive secretion of ECM (Travers et al., 2016). Transcriptome changes are required for fibroblast transformation. Myofibroblasts express numerous contractility-related genes, 
including Acta2 and Tagln, and secrete abundant ECM components, such as collagen I and III, fibronectin 1, tenascin- $\mathrm{C}$, periostin, and matrix metalloproteinases (Travers et al., 2016). However, unlike other tissues, abundant ECM deposition does not stimulate cardiomyocytes to repair injury due to the terminal differentiation of cardiomyocytes. Additionally, the transformed myofibroblasts do not return to quiescent fibroblasts. Notably, after acute injury, the activated cardiac fibroblasts not only secret excessive ECM in the damaged site, but also produce large amount of ECM in remote healthy tissue, which reduces tissue compliance and progress into overt heart failure (Travers et al., 2016). Therefore, although early- and short-term cardiac fibrosis adapts to heart remodeling, persistent fibrosis is still detrimental to cardiac function.

\subsubsection{Small Mothers Against Decapentaplegic Transcription Factors}

Small mothers against the decapentaplegic (SMAD) is a critical transcription factor family controlling multiple genes expression in myofibroblast, such as Col1a, Acta2, and Tagln (Lighthouse and Small, 2016). The SMAD family consists of three subtypes with different transcriptional functions: the activating SMADs ( 1 , $2,3,5$ and 8), the inhibitory SMADs (6 and 7), and the cofactor SMAD4. Additionally, among these activating SMADs, they are activated by different signals. Briefly, SMADs 1, 5 and 8 are generally activated by bone morphogenetic proteins while SMADs 2 and 3 are often referred to transforming growth factor- $\beta$ (Hanna et al., 2021). In conical TGF- $\beta$ pathway, activation of either BMP or TGF- $\beta$ leads to SMADs activation and fibroblast activation through T $\beta$ RI. Notably, some researchers reported that non-conical TGF- $\beta$ pathway also played critical roles in activating SMADs and ECM synthesis through other signaling molecular but not T $\beta \mathrm{RI}$, such as Rhoassociated protein kinase (Burch et al., 2011; Kamato et al., 2013). Directly loss-of-function or gain-of-function studies also revealed the important regulating function of SMADs in fibrosis and ECM synthesis. In SMAD3-deficient mice, cardiac fibroblasts produce less collagen and included fewer activated fibroblasts characterized by Acta2 positivity than wild-type mice (Dobaczewski et al., 2010). Furthermore, cardiac remodeling induced by myocardial infarction (MI) and diabetes mellitus are attenuated by the loss of SMAD3 in mice (Dobaczewski et al., 2010). Recently, Zhou et al. (2019) found that the Notch signaling pathway antagonizes SMAD3 activation and inhibits fibroblast transformation. Similar to SMAD3, cardiac fibroblastspecific loss of SMAD2 transiently ameliorates postinfarctioninduced cardiac hypertrophy with preserved ventricular function (Huang et al., 2019). However, cardiac fibroblast-specific knockout of SMAD2 does not alter collagen expression (Huang et al., 2019). Additionally, SMAD3, but not SMAD2, regulates the formation of organized myofibroblast arrays and controls integrin and GTPase RhoA expression (Huang et al., 2019). Notably, inhibitory SMAD7 is downregulated in hearts of rats with MI (Wang et al., 2007). Several SMAD7-related noncoding RNAs have been identified in cardiac fibroblasts, such as miR-21, miR-216a, and lncRNA-Ang362, which promote cardiac fibrosis in various animal models by suppressing SMAD7 expression (Yuan et al., 2017; Tao et al., 2019; Chen et al., 2020).

\subsubsection{Myocardin-Related Transcription Factor}

Recent studies have revealed the predominant function of myocardin-related transcription factor (MRTF) in cardiac fibrosis, which is encoding by megakaryoblastic leukemia genes. In the quiescent state, MRTF is broadly expressed and sequestered in the cytoplasm by binding G-actin. Stress stimuli, including hypoxia, inflammation, and ROS, induce the polymerization of F-actin and alterations in these connections, permitting the nuclear translocation of MRTF. Subsequently, the translocated MRTF forms a complex with SRF to bind CArG elements in the promoters of several genes related to fibrosis. Blockade of MRTF-A reduces scar and fibrosis formation after MI or angiotensin II treatment in mice (Small et al., 2010; Francisco et al., 2020). Additionally, MRTF-A-null mice display decreased expression of genes involved in extracellular matrix production and smooth muscle cell differentiation, especially Colla2 expression (Small et al., 2010). Therefore, approaches targeting MRTF appear to be a good and new therapeutic method for treating cardiac injury. Indeed, many small-molecule MRTF inhibitors have been exploited and shown good performance in treating local fibrosis in various tissues, but not in the heart (Yu-Wai-Man et al., 2017). The strategy of treating cardiac fibrosis by targeting MRTF is worth exploring.

\subsubsection{Nuclear Factor of Activated T-Cells}

Similar to cardiomyocytes, the calcineurin/NFAT signaling pathway is critical for fibroblasts. The dephosphorylation and translocation of NFAT triggers fibrotic gene expression in cardiac fibroblasts, including Col3 and MRTF-A (Herum et al., 2013). In pressure-overloaded mice, upregulated calcineurin/NFAT contributes to cardiac fibrosis formation (Wang et al., 2016). Interestingly, regardless of the pharmacological inhibition of calcineurin by cyclosporin A or mutations in calcineurin, NFAT translocation is downregulated and cardiac fibrosis is ameliorated in PE-treated primary rat cardiac fibroblasts (Wang et al., 2016). Currently, an increasing number of researchers have focused on signaling upstream of calcineurin/ NFAT through a $\mathrm{Ca}^{2+}$ transporter called transient receptor potential (TRP). TRPA1 expression is required for TGF- $\beta$ induced fibroblast activation in a calcineurin/NFATc3dependent manner (Li S et al., 2019). Similarly, in addition to TRPC3, TRPC6, TRPM6, TRPM7, TRPA1, TRPV3 and TRPV4 have been reported to directly participate in cardiac fibrosis progression by regulating the intercellular $\mathrm{Ca}^{2+}$ concentration (Inoue et al., 2019).

\subsubsection{SRF}

In cardiac fibroblasts, function of SRF have been extensively studied. SRF activates cardiac fibroblasts via promoting several genes transcription, such as Acta2 and Col1 (Bretherton et al., 2020). As mentioned above, MRTF is a key factor for SRF transcriptional activation. Cleaved rho-associated coiled-coil protein kinase 1 isoform ( $\mathrm{N}$-terminal part) promotes SRF to initiate the targeted gene, TGF- $\beta 1$, transcription by releasing 
MRTF from G-actin, which causes cardiac fibroblasts activation subsequently (Yang et al., 2012). Besides Rho/ MRTF pathway, MAPK/Extracellular regulated protein kinases (ERK) 1/2 pathway also participates the activation of SRF. The ERK1/2-activated SRF promotes cellular inhibitor of apoptosis 2 expression in cardiac fibroblast. Cell cycle of cardiac fibroblasts is also regulated by SRF activation. The activated SRF up-regulated S-phase kinase-associated protein 2 expression, which facilitates post-translational degradation of p27, a cyclin-dependent kinase inhibitor arresting cell cycle, to promote G1-S transition and cardiac fibroblast proliferation (Titus et al., 2020). It is still unclear how ERK1/2 influence SRF activation in cardiac fibroblasts. However, it has been reported that this activation effect is mediated by ternary complex factors in embryo fibroblast, which competitively inhibits MRTF binding with SRF (Gualdrini et al., 2016). Similar mechanism may happen in cardiac fibroblasts, further explorations are needed.

\subsubsection{Transcription Factor 21}

Cardiac fibroblasts are thought to be predominantly derived from the epicardial cells (Deb and Ubil, 2014). Through a process called epithelial-to-mesenchymal transition (EMT), some epicardial cells migrates into the myocardium and differentiate into coronary vascular smooth muscle cells or cardiac fibroblasts. During EMT process, transcription factor 21 (TCF21) express in epicardial cells. Genetic lineage tracing study showed that TCF21 specifically expresses in cardiac fibroblasts lineage, which is essential for the cell fate determination of cardiac fibroblasts in development and remains active in adult mice (Ma et al., 2017). Therefore, TCF21 is often referred as a biomarker for tracing cardiac fibroblasts in developmental and disease study. Additionally, the inducible TCF $21^{\mathrm{mERCremER}}$ transgenic mice, which specifically express Cre recombinase in cardiac fibroblasts after treatment with tamoxifen, represents as a powerful mice line for studying cardiac fibroblasts (Travers et al., 2016). After acute injury, TCF21-expressing epicardial cells undergo EMT to generate cardiac fibroblasts, which express collagen and contribute to pro-fibrotic repair response, indicating TCF21 expression play critical role in the cardiac fibrosis (Acharya et al., 2012; Deb and Ubil, 2014).

\section{REFERENCES}

Acharya, A., Baek, S. T., Huang, G., Eskiocak, B., Goetsch, S., Sung, C. Y., et al. (2012). The bHLH Transcription Factor Tcf21 Is Required for Lineage-specific EMT of Cardiac Fibroblast Progenitors. Development 139 (12), 2139-2149. doi:10.1242/dev.079970

Ajith, T. A., and Jayakumar, T. G. (2016). Peroxisome Proliferator-Activated Receptors in Cardiac Energy Metabolism and Cardiovascular Disease. Clin. Exp. Pharmacol. Physiol. 43 (7), 649-658. doi:10.1111/1440-1681.12579

Barlaka, E., Görbe, A., Gáspár, R., Pálóczi, J., Ferdinandy, P., and Lazou, A. (2015). Activation of PPAR $\beta / \delta$ Protects Cardiac Myocytes from Oxidative StressInduced Apoptosis by Suppressing Generation of Reactive Oxygen/nitrogen Species and Expression of Matrix Metalloproteinases. Pharmacol. Res. 95-96, 102-110. doi:10.1016/j.phrs.2015.03.008

\section{CONCLUSION AND PERSPECTIVE}

Many transcription factors, their target genes, protein interactions and biological functions have been recognized in the heart. Classically, transcription factors govern the expression of several critical proteins to influence related cellular processes. In addition to the canonical function of transcription factors in controlling functional protein expression, emerging evidence proves that they also play critical roles in epigenetics in the heart. Therefore, the recognition of transcription factors would help us to explore the cardiac disease process. Indeed, with the help of next-generation sequencing technology, patients' genetic patterns have been characterized gradually. Mutations in multiple genes have been found to be directly associated with diseases, including cardiovascular diseases. Transcription factors, as directly upstream molecules regulating gene expression, might be an effective target for developing new cardiovascular drugs. In fact, some molecules from natural products have been proven to target transcription factors and treat heart diseases. However, few drugs ultimately enter the clinic and save patients' lives. In this article, we summarized some extensively studied transcription factors involved in various cellular pathways in the heart, such as fetal reprogramming, energy metabolism, apoptosis, autophagy and fibrosis, and promise to provide insights for the further exploration of the mechanism and drug research for heart diseases in the future.

\section{AUTHOR CONTRIBUTIONS}

$\mathrm{J}-\mathrm{HH}$ wrote and drafted of the article. H-GZ revised the manuscript critically. All authors contributed to the article and approved the submitted version.

\section{FUNDING}

This work was supported by grant from the Special project for promoting scientific and technological innovation capability: Frontier exploration project, Third Military Medical University (No. 2019XQY05).

Bretherton, R., Bugg, D., Olszewski, E., and Davis, J. (2020). Regulators of Cardiac Fibroblast Cell State. Matrix Biol. 91-92, 117-135. doi:10.1016/j.matbio.2020.04.002

Burch, M. L., Zheng, W., and Little, P. J. (2011). Smad Linker Region Phosphorylation in the Regulation of Extracellular Matrix Synthesis. Cell. Mol. Life Sci. 68 (1), 97-107. doi:10.1007/s00018-010-0514-4

Burkart, E. M., Sambandam, N., Han, X., Gross, R. W., Courtois, M., Gierasch, C. M., et al. (2007). Nuclear Receptors PPAR $\beta / \delta$ and PPARa Direct Distinct Metabolic Regulatory Programs in the Mouse Heart. J. Clin. Invest. 117 (12), 3930-3939. doi:10.1172/jci32578

Caragnano, A., Aleksova, A., Bulfoni, M., Cervellin, C., Rolle, I. G., Veneziano, C., et al. (2019). Autophagy and Inflammasome Activation in Dilated Cardiomyopathy. J. Clin. Med. 8 (10), 1519. doi:10.3390/jcm8101519

Carroll, C. J., Sayan, B. S., Bailey, S. G., McCormick, J., Stephanou, A., Latchman, D. S., et al. (2013). Regulation of Myocardial Interleukin-6 Expression by P53 and STAT1. J. Interferon Cytokine Res. 33 (9), 542-548. doi:10.1089/jir.2012.0165 
Chang, J., Wei, L., Otani, T., Youker, K. A., Entman, M. L., and Schwartz, R. J. (2003). Inhibitory Cardiac Transcription Factor, SRF-N, Is Generated by Caspase 3 Cleavage in Human Heart Failure and Attenuated by Ventricular Unloading. Circulation 108 (4), 407-413. doi:10.1161/01.CIR.0000084502. 02147.83

Chen, J. N., and Fishman, M. C. (1996). Zebrafish Tinman Homolog Demarcates the Heart Field and Initiates Myocardial Differentiation. Development 122 (12), 3809-3816. doi:10.1242/dev.122.12.3809

Chen, R., Liang, F., Morimoto, S., Li, Q., Moriya, J., Yamakawa, J., et al. (2010). The Effects of a PPARalpha Agonist on Myocardial Damage in Obese Diabetic Mice with Heart Failure. Int. Heart J. 51 (3), 199-206. doi:10.1536/ihj.51.199

Chen, Z. C., Yu, B. C., Chen, L. J., and Cheng, J. T. (2013). Increase of Peroxisome Proliferator-Activated Receptor $\delta$ (PPAR $\delta$ ) by Digoxin to Improve Lipid Metabolism in the Heart of Diabetic Rats. Horm. Metab. Res. 45 (5), 364-371. doi:10.1055/s-0032-1330021

Chen, C., Ponnusamy, M., Liu, C., Gao, J., Wang, K., and Li, P. (2017). MicroRNA as a Therapeutic Target in Cardiac Remodeling. Biomed. Res. Int. 2017, 1278436. doi:10.1155/2017/1278436

Chen, Q., Thompson, J., Hu, Y., Das, A., and Lesnefsky, E. J. (2019). Cardiac Specific Knockout of P53 Decreases ER Stress-Induced Mitochondrial Damage. Front. Cardiovasc. Med. 6, 10. doi:10.3389/fcvm.2019.00010

Chen, G., Huang, S., Song, F., Zhou, Y., and He, X. (2020). Lnc-Ang362 Is a Profibrotic Long Non-coding RNA Promoting Cardiac Fibrosis after Myocardial Infarction by Suppressing Smad7. Arch. Biochem. Biophys. 685, 108354. doi:10. 1016/j.abb.2020.108354

Clapham, K. R., Singh, I., Capuano, I. S., Rajagopal, S., and Chun, H. J. (2019). MEF2 and the Right Ventricle: From Development to Disease. Front. Cardiovasc. Med. 6, 29. doi:10.3389/fcvm.2019.00029

Czibik, G., Wu, Z., Berne, G. P., Tarkka, M., Vaage, J., Laurikka, J., et al. (2008). Human Adaptation to Ischemia by Preconditioning or Unstable Angina: Involvement of Nuclear Factor Kappa B, but Not Hypoxia-Inducible Factor 1 Alpha in the Heart. Eur. J. Cardiothorac. Surg. 34 (5), 976-984. doi:10.1016/j. ejcts.2008.07.066

Deb, A., and Ubil, E. (2014). Cardiac Fibroblast in Development and Wound Healing. J. Mol. Cel. Cardiol. 70, 47-55. doi:10.1016/j.yjmcc.2014.02.017

Desjardins, C. A., and Naya, F. J. (2016). The Function of the MEF2 Family of Transcription Factors in Cardiac Development, Cardiogenomics, and Direct Reprogramming. J. Cardiovasc. Dev. Dis. 3 (3), 26. doi:10.3390/jcdd3030026

Diedrichs, H., Chi, M., Boelck, B., Mehlhorn, U., Mehlhorm, U., and Schwinger, R. H. (2004). Increased Regulatory Activity of the Calcineurin/NFAT Pathway in Human Heart Failure. Eur. J. Heart Fail. 6 (1), 3-9. doi:10.1016/j.ejheart.2003. 07.007

Dobaczewski, M., Bujak, M., Li, N., Gonzalez-Quesada, C., Mendoza, L. H., Wang, X. F., et al. (2010). Smad3 Signaling Critically Regulates Fibroblast Phenotype and Function in Healing Myocardial Infarction. Circ. Res. 107 (3), 418-428. doi:10.1161/CIRCRESAHA.109.216101

Dolcet, X., Llobet, D., Pallares, J., and Matias-Guiu, X. (2005). NF-kB in Development and Progression of Human Cancer. Virchows Arch. 446 (5), 475-482. doi:10.1007/s00428-005-1264-9

Duan, S. Z., Ivashchenko, C. Y., Russell, M. W., Milstone, D. S., and Mortensen, R. M. (2005). Cardiomyocyte-specific Knockout and Agonist of Peroxisome Proliferator-Activated Receptor-Gamma Both Induce Cardiac Hypertrophy in Mice. Circ. Res. 97 (4), 372-379. doi:10.1161/01.RES.0000179226.34112.6d

Eid, R. A., Alkhateeb, M. A., Eleawa, S., Al-Hashem, F. H., Al-Shraim, M., El-Kott, A. F., et al. (2018). Cardioprotective Effect of Ghrelin against Myocardial Infarction-Induced Left Ventricular Injury via Inhibition of SOCS3 and Activation of JAK2/STAT3 Signaling. Basic Res. Cardiol. 113 (2), 13. doi:10. 1007/s00395-018-0671-4

Fan, Y., Liu, J., Miao, J., Zhang, X., Yan, Y., Bai, L., et al. (2021). Anti-inflammatory Activity of the Tongmai Yangxin Pill in the Treatment of Coronary Heart Disease Is Associated with Estrogen Receptor and NF- $\kappa B$ Signaling Pathway. J. Ethnopharmacol. 276, 114106. doi:10.1016/j.jep.2021.114106

Ferdous, A., Battiprolu, P. K., Ni, Y. G., Rothermel, B. A., and Hill, J. A. (2010). FoxO, Autophagy, and Cardiac Remodeling. J. Cardiovasc. Transl. Res. 3 (4), 355-364. doi:10.1007/s12265-010-9200-z

Finck, B. N., Lehman, J. J., Leone, T. C., Welch, M. J., Bennett, M. J., Kovacs, A., et al. (2002). The Cardiac Phenotype Induced by PPARalpha Overexpression
Mimics that Caused by Diabetes Mellitus. J. Clin. Invest. 109 (1), 121-130. doi:10.1172/JCI14080

Francisco, J., Zhang, Y., Jeong, J. I., Mizushima, W., Ikeda, S., Ivessa, A., et al. (2020). Blockade of Fibroblast YAP Attenuates Cardiac Fibrosis and Dysfunction through MRTF-A Inhibition. JACC Basic Transl. Sci. 5 (9), 931-945. doi:10.1016/j.jacbts.2020.07.009

Frantz, S., Hu, K., Bayer, B., Gerondakis, S., Strotmann, J., Adamek, A., et al. (2006). Absence of NF-kappaB Subunit P50 Improves Heart Failure after Myocardial Infarction. FASEB J. 20 (11), 1918-1920. doi:10.1096/fj.05-5133fje

Galasso, G., De Rosa, R., Piscione, F., Iaccarino, G., Vosa, C., Sorriento, D., et al. (2010). Myocardial Expression of FOXO3a-Atrogin-1 Pathway in Human Heart Failure. Eur. J. Heart Fail. 12 (12), 1290-1296. doi:10.1093/eurjhf/hfq102

Gogna, R., Madan, E., Khan, M., Pati, U., and Kuppusamy, P. (2013). p53's Choice of Myocardial Death or Survival: Oxygen Protects Infarct Myocardium by Recruiting P53 on NOS3 Promoter through Regulation of P53-Lys(118) Acetylation. EMBO Mol. Med. 5 (11), 1662-1683. doi:10.1002/emmm. 201202055

Gualdrini, F., Esnault, C., Horswell, S., Stewart, A., Matthews, N., and Treisman, R. (2016). SRF Co-factors Control the Balance between Cell Proliferation and Contractility. Mol. Cel 64 (6), 1048-1061. doi:10.1016/j.molcel.2016.10.016

Hamid, T., Guo, S. Z., Kingery, J. R., Xiang, X., Dawn, B., and Prabhu, S. D. (2011). Cardiomyocyte NF- $\kappa$ B P65 Promotes Adverse Remodelling, Apoptosis, and Endoplasmic Reticulum Stress in Heart Failure. Cardiovasc. Res. 89 (1), 129-138. doi:10.1093/cvr/cvq274

Han, M., Chen, X.-C., Sun, M.-H., Gai, M.-T., Yang, Y.-N., Gao, X.-M., et al. (2020). Overexpression of I $\mathrm{KB} \alpha$ in Cardiomyocytes Alleviates Hydrogen PeroxideInduced Apoptosis and Autophagy by Inhibiting NF- $\mathrm{kB}$ Activation. Lipids Health Dis. 19 (1), 150. doi:10.1186/s12944-020-01327-2

Hanna, A., Humeres, C., and Frangogiannis, N. G. (2021). The Role of Smad Signaling Cascades in Cardiac Fibrosis. Cell. Signal. 77, 109826. doi:10.1016/j. cellsig.2020.109826

Harhous, Z., Booz, G. W., Ovize, M., Bidaux, G., and Kurdi, M. (2019). An Update on the Multifaceted Roles of STAT3 in the Heart. Front. Cardiovasc. Med. 6, 150. doi:10.3389/fcvm.2019.00150

Hariharan, N., Maejima, Y., Nakae, J., Paik, J., Depinho, R. A., and Sadoshima, J. (2010). Deacetylation of FoxO by Sirtl Plays an Essential Role in Mediating Starvation-Induced Autophagy in Cardiac Myocytes. Circ. Res. 107 (12), 1470-1482. doi:10.1161/CIRCRESAHA.110.227371

Herum, K. M., Lunde, I. G., Skrbic, B., Florholmen, G., Behmen, D., Sjaastad, I., et al. (2013). Syndecan-4 Signaling via NFAT Regulates Extracellular Matrix Production and Cardiac Myofibroblast Differentiation in Response to Mechanical Stress. J. Mol. Cel. Cardiol. 54, 73-81. doi:10.1016/j.yjmcc.2012. 11.006

Hilfiker-Kleiner, D., Hilfiker, A., Fuchs, M., Kaminski, K., Schaefer, A., Schieffer, B., et al. (2004). Signal Transducer and Activator of Transcription 3 Is Required for Myocardial Capillary Growth, Control of Interstitial Matrix Deposition, and Heart protection from Ischemic Injury. Circ. Res. 95 (2), 187-195. doi:10.1161/ 01.RES.0000134921.50377.61

Huang, S., Chen, B., Su, Y., Alex, L., Humeres, C., Shinde, A. V., et al. (2019). Distinct Roles of Myofibroblast-specific Smad2 and Smad3 Signaling in Repair and Remodeling of the Infarcted Heart. J. Mol. Cel. Cardiol. 132, 84-97. doi:10. 1016/j.yjmcc.2019.05.006

Imam, F., Al-Harbi, N. O., Al-Harbi, M. M., Ansari, M. A., Al-Asmari, A. F., Ansari, M. N., et al. (2018). Apremilast Prevent Doxorubicin-Induced Apoptosis and Inflammation in Heart through Inhibition of Oxidative Stress Mediated Activation of NF- $\mathrm{kB}$ Signaling Pathways. Pharmacol. Rep. 70 (5), 993-1000. doi:10.1016/j.pharep.2018.03.009

Ingles, J., Goldstein, J., Thaxton, C., Caleshu, C., Corty, E. W., Crowley, S. B., et al. (2019). Evaluating the Clinical Validity of Hypertrophic Cardiomyopathy Genes. Circ. Genom. Precis. Med. 12 (2), e002460. doi:10.1161/CIRCGEN. 119.002460

Inoue, R., Kurahara, L. H., and Hiraishi, K. (2019). TRP Channels in Cardiac and Intestinal Fibrosis. Semin. Cel Dev. Biol. 94, 40-49. doi:10.1016/j.semcdb.2018. 11.002

Kaimoto, S., Hoshino, A., Ariyoshi, M., Okawa, Y., Tateishi, S., Ono, K., et al. (2017). Activation of PPAR- $\alpha$ in the Early Stage of Heart Failure Maintained Myocardial Function and Energetics in Pressure-Overload Heart Failure. Am. 
J. Physiol. Heart Circ. Physiol. 312 (2), H305-H313. doi:10.1152/ajpheart.00553. 2016

Kamato, D., Burch, M. L., Piva, T. J., Rezaei, H. B., Rostam, M. A., Xu, S., et al. (2013). Transforming Growth Factor- $\beta$ Signalling: Role and Consequences of Smad Linker Region Phosphorylation. Cel. Signal. 25 (10), 2017-2024. doi:10. 1016/j.cellsig.2013.06.001

Karwi, Q. G., Uddin, G. M., Ho, K. L., and Lopaschuk, G. D. (2018). Loss of Metabolic Flexibility in the Failing Heart. Front. Cardiovasc. Med. 5, 68. doi:10. $3389 /$ fcvm. 2018.00068

Khuchua, Z., Glukhov, A. I., Strauss, A. W., and Javadov, S. (2018). Elucidating the Beneficial Role of PPAR Agonists in Cardiac Diseases. Int. J. Mol. Sci. 19 (11), 3464. doi:10.3390/ijms19113464

Knight, R. A., Scarabelli, T. M., and Stephanou, A. (2012). STAT Transcription in the Ischemic Heart. JAKSTAT 1 (2), 111-117. doi:10.4161/jkst.20078

Kong, P., Christia, P., and Frangogiannis, N. G. (2014). The Pathogenesis of Cardiac Fibrosis. Cel. Mol. Life Sci. 71 (4), 549-574. doi:10.1007/s00018-013$1349-6$

Kratsios, P., Huth, M., Temmerman, L., Salimova, E., Al Banchaabouchi, M., Sgoifo, A., et al. (2010). Antioxidant Amelioration of Dilated Cardiomyopathy Caused by Conditional Deletion of NEMO/IKKgamma in Cardiomyocytes. Circ. Res. 106 (1), 133-144. doi:10.1161/CIRCRESAHA.109.202200

Li, F., Lang, F., Zhang, H., Xu, L., Wang, Y., and Hao, E. (2016). Role of TFEB Mediated Autophagy, Oxidative Stress, Inflammation, and Cell Death in Endotoxin Induced Myocardial Toxicity of Young and Aged Mice. Oxid. Med. Cel. Longev. 2016, 5380319. doi:10.1155/2016/5380319

Li S, S., Sun, X., Wu, H., Yu, P., Wang, X., Jiang, Z., et al. (2019). TRPA1 Promotes Cardiac Myofibroblast Transdifferentiation after Myocardial Infarction Injury via the Calcineurin-NFAT-DYRK1A Signaling Pathway. Oxid. Med. Cel. Longev. 2019, 6408352. doi:10.1155/2019/6408352

Li X, X., Zhao, J., Geng, J., Chen, F., Wei, Z., Liu, C., et al. (2019). Long Non-coding RNA MEG3 Knockdown Attenuates Endoplasmic Reticulum Stress-Mediated Apoptosis by Targeting P53 Following Myocardial Infarction. J. Cel. Mol. Med. 23 (12), 8369-8380. doi:10.1111/jcmm.14714

Li, J., Tan, Y., Passariello, C. L., Martinez, E. C., Kritzer, M. D., Li, X., et al. (2020). Signalosome-Regulated Serum Response Factor Phosphorylation Determining Myocyte Growth in Width versus Length as a Therapeutic Target for Heart Failure. Circulation 142 (22), 2138-2154. doi:10.1161/CIRCULATIONAHA. 119.044805

Liang, Q., De Windt, L. J., Witt, S. A., Kimball, T. R., Markham, B. E., and Molkentin, J. D. (2001). The Transcription Factors GATA4 and GATA6 Regulate Cardiomyocyte Hypertrophy In Vitro and In Vivo. J. Biol. Chem. 276 (32), 30245-30253. doi:10.1074/jbc.M102174200

Lighthouse, J. K., and Small, E. M. (2016). Transcriptional Control of Cardiac Fibroblast Plasticity. J. Mol. Cel. Cardiol. 91, 52-60. doi:10.1016/j.yjmcc.2015. 12.016

Lints, T. J., Parsons, L. M., Hartley, L., Lyons, I., and Harvey, R. P. (1993). Nkx-2.5: a Novel Murine Homeobox Gene Expressed in Early Heart Progenitor Cells and Their Myogenic Descendants. Development 119 (2), 969-431. doi:10.1242/dev. 119.2.419

Liu, W., Liu, Y., Zhang, Y., Zhu, X., Zhang, R., Guan, L., et al. (2015). MicroRNA150 Protects against Pressure Overload-Induced Cardiac Hypertrophy. J. Cel. Biochem. 116 (10), 2166-2176. doi:10.1002/jcb.25057

Liu, X., Yu, Z., Huang, X., Gao, Y., Wang, X., Gu, J., et al. (2016). Peroxisome Proliferator-Activated Receptor $\gamma$ (PPAR $\gamma$ ) Mediates the Protective Effect of Quercetin against Myocardial Ischemia-Reperfusion Injury via Suppressing the NF-kB Pathway. Am. J. Transl. Res. 8 (12), 5169-5186.

Liu, Y., Chen, X., and Zhang, H. G. (2021). Editorial: Cardiac Hypertrophy: From Compensation to Decompensation and Pharmacological Interventions. Front. Pharmacol. 12, 665936. doi:10.3389/fphar.2021.665936

Lu, D., Liu, J., Jiao, J., Long, B., Li, Q., Tan, W., et al. (2013). Transcription Factor Foxo3a Prevents Apoptosis by Regulating Calcium through the Apoptosis Repressor with Caspase Recruitment Domain. J. Biol. Chem. 288 (12), 8491-8504. doi:10.1074/jbc.M112.442061

Luo, M., Yan, D., Sun, Q., Tao, J., Xu, L., Sun, H., et al. (2020). Ginsenoside Rg1 Attenuates Cardiomyocyte Apoptosis and Inflammation via the TLR4/NF-kB/ NLRP3 Pathway. J. Cel. Biochem. 121 (4), 2994-3004. doi:10.1002/jcb.29556

Luo, Y., Jiang, N., May, H. I., Luo, X., Ferdous, A., Schiattarella, G. G., et al. (2021). Cooperative Binding of ETS2 and NFAT Links Erk1/2 and Calcineurin
Signaling in the Pathogenesis of Cardiac Hypertrophy. Circulation 144 (1), 34-51. doi:10.1161/CIRCULATIONAHA.120.052384

Ma, Y., Iyer, R. P., Jung, M., Czubryt, M. P., and Lindsey, M. L. (2017). Cardiac Fibroblast Activation Post-Myocardial Infarction: Current Knowledge Gaps. Trends Pharmacol. Sci. 38 (5), 448-458. doi:10.1016/j.tips.2017.03.001

Mangali, S., Bhat, A., Udumula, M. P., Dhar, I., Sriram, D., and Dhar, A. (2019). Inhibition of Protein Kinase $\mathrm{R}$ Protects against Palmitic Acid-Induced Inflammation, Oxidative Stress, and Apoptosis through the JNK/NF-kB/ NLRP3 Pathway in Cultured H9C2 Cardiomyocytes. J. Cel. Biochem. 120 (3), 3651-3663. doi:10.1002/jcb.27643

Mehrabi, M. R., Haslmayer, P., Humpeler, S., Strauss-Blasche, G., Marktl, W., Tamaddon, F., et al. (2003). Quantitative Analysis of Peroxisome ProliferatorActivated Receptor Gamma (PPARgamma) Expression in Arteries and Hearts of Patients with Ischaemic or Dilated Cardiomyopathy. Eur. J. Heart Fail. 5 (6), 733-739. doi:10.1016/s1388-9842(03)00148-x

Men, H., Cai, H., Cheng, Q., Zhou, W., Wang, X., Huang, S., et al. (2021). The Regulatory Roles of P53 in Cardiovascular Health and Disease. Cel. Mol. Life Sci. 78 (5), 2001-2018. doi:10.1007/s00018-020-03694-6

Molkentin, J. D., Lu, J. R., Antos, C. L., Markham, B., Richardson, J., Robbins, J., et al. (1998). A Calcineurin-dependent Transcriptional Pathway for Cardiac Hypertrophy. Cell 93 (2), 215-228. doi:10.1016/s0092-8674(00)81573-1

Nelson, T. J., Balza, R., Jr., Xiao, Q., and Misra, R. P. (2005). SRF-dependent Gene Expression in Isolated Cardiomyocytes: Regulation of Genes Involved in Cardiac Hypertrophy. J. Mol. Cel. Cardiol. 39 (3), 479-489. doi:10.1016/j. yjmcc.2005.05.004

Ng, D. C., Court, N. W., dos Remedios, C. G., and Bogoyevitch, M. A. (2003). Activation of Signal Transducer and Activator of Transcription (STAT) Pathways in Failing Human Hearts. Cardiovasc. Res. 57 (2), 333-346. doi:10.1016/s0008-6363(02)00664-8

Niu, Z., Yu, W., Zhang, S. X., Barron, M., Belaguli, N. S., Schneider, M. D., et al. (2005). Conditional Mutagenesis of the Murine Serum Response Factor Gene Blocks Cardiogenesis and the Transcription of Downstream Gene Targets. J. Biol. Chem. 280 (37), 32531-32538. doi:10.1074/jbc.M501372200

Oka, S., Zhai, P., Yamamoto, T., Ikeda, Y., Byun, J., Hsu, C. P., et al. (2015). Peroxisome Proliferator Activated Receptor- $\alpha$ Association with Silent Information Regulator 1 Suppresses Cardiac Fatty Acid Metabolism in the Failing Heart. Circ. Heart Fail. 8 (6), 1123-1132. doi:10.1161/ CIRCHEARTFAILURE.115.002216

Oldfield, C. J., Duhamel, T. A., and Dhalla, N. S. (2020). Mechanisms for the Transition from Physiological to Pathological Cardiac Hypertrophy. Can. J. Physiol. Pharmacol. 98 (2), 74-84. doi:10.1139/cjpp-2019-0566

Oshima, Y., Fujio, Y., Nakanishi, T., Itoh, N., Yamamoto, Y., Negoro, S., et al. (2005). STAT3 Mediates Cardioprotection against Ischemia/reperfusion Injury through Metallothionein Induction in the Heart. Cardiovasc. Res. 65 (2), 428-435. doi:10.1016/j.cardiores.2004.10.021

Pan, B., Zhang, H., Cui, T., and Wang, X. (2017). TFEB Activation Protects against Cardiac Proteotoxicity via Increasing Autophagic Flux. J. Mol. Cel. Cardiol. 113, 51-62. doi:10.1016/j.yjmcc.2017.10.003

Parlakian, A., Charvet, C., Escoubet, B., Mericskay, M., Molkentin, J. D., GaryBobo, G., et al. (2005). Temporally Controlled Onset of Dilated Cardiomyopathy through Disruption of the SRF Gene in Adult Heart. Circulation 112 (19), 2930-2939. doi:10.1161/CIRCULATIONAHA.105. 533778

Raut, S. K., Singh, G. B., Rastogi, B., Saikia, U. N., Mittal, A., Dogra, N., et al. (2016). miR-30c and miR-181a Synergistically Modulate P53-P21 Pathway in Diabetes Induced Cardiac Hypertrophy. Mol. Cel. Biochem. 417 (1-2), 191-203. doi:10. 1007/s11010-016-2729-7

Schubert, W., Yang, X. Y., Yang, T. T., Factor, S. M., Lisanti, M. P., Molkentin, J. D., et al. (2003). Requirement of Transcription Factor NFAT in Developing Atrial Myocardium. J. Cel. Biol. 161 (5), 861-874. doi:10.1083/jcb.200301058

Schüttler, D., Clauss, S., Weckbach, L. T., and Brunner, S. (2019). Molecular Mechanisms of Cardiac Remodeling and Regeneration in Physical Exercise. Cells 8 (10), 1128. doi:10.3390/cells8101128

Sciarretta, S., Maejima, Y., Zablocki, D., and Sadoshima, J. (2018). The Role of Autophagy in the Heart. Annu. Rev. Physiol. 80, 1-26. doi:10.1146/annurevphysiol-021317-121427

Sengupta, A., Molkentin, J. D., Paik, J. H., DePinho, R. A., and Yutzey, K. E. (2011). FoxO Transcription Factors Promote Cardiomyocyte Survival upon Induction 
of Oxidative Stress. J. Biol. Chem. 286 (9), 7468-7478. doi:10.1074/jbc.M110. 179242

Settembre, C., and Ballabio, A. (2011). TFEB Regulates Autophagy: an Integrated Coordination of Cellular Degradation and Recycling Processes. Autophagy 7 (11), 1379-1381. doi:10.4161/auto.7.11.17166

Shimizu, I., and Minamino, T. (2016). Physiological and Pathological Cardiac Hypertrophy. J. Mol. Cel. Cardiol. 97, 245-262. doi:10.1016/j.yjmcc.2016.06.001

Shiojima, I., Komuro, I., Oka, T., Hiroi, Y., Mizuno, T., Takimoto, E., et al. (1999). Context-dependent Transcriptional Cooperation Mediated by Cardiac Transcription Factors Csx/Nkx-2.5 and GATA-4. J. Biol. Chem. 274 (12), 8231-8239. doi:10.1074/jbc.274.12.8231

Small, E. M., Thatcher, J. E., Sutherland, L. B., Kinoshita, H., Gerard, R. D., Richardson, J. A., et al. (2010). Myocardin-related Transcription Factor-A Controls Myofibroblast Activation and Fibrosis in Response to Myocardial Infarction. Circ. Res. 107 (2), 294-304. doi:10.1161/CIRCRESAHA.110.223172

Son, N. H., Park, T. S., Yamashita, H., Yokoyama, M., Huggins, L. A., Okajima, K., et al. (2007). Cardiomyocyte Expression of PPARgamma Leads to Cardiac Dysfunction in Mice. J. Clin. Invest. 117 (10), 2791-2801. doi:10.1172/JCI30335

Son, N. H., Yu, S., Tuinei, J., Arai, K., Hamai, H., Homma, S., et al. (2010). PPAR $\gamma$ induced Cardiolipotoxicity in Mice Is Ameliorated by PPARa Deficiency Despite Increases in Fatty Acid Oxidation. J. Clin. Invest. 120 (10), 3443-3454. doi:10.1172/JCI40905

Song, H., Conte, J. V., Jr., Foster, A. H., McLaughlin, J. S., and Wei, C. (1999). Increased P53 Protein Expression in Human Failing Myocardium. J. Heart Lung Transpl. 18 (8), 744-749. doi:10.1016/s1053-2498(98)00039-4

Tao, J., Wang, J., Li, C., Wang, W., Yu, H., Liu, J., et al. (2019). MiR-216a Accelerates Proliferation and Fibrogenesis via Targeting PTEN and SMAD7 in Human Cardiac Fibroblasts. Cardiovasc. Diagn. Ther. 9 (6), 535-544. doi:10. 21037/cdt.2019.11.06

Teringova, E., and Tousek, P. (2017). Apoptosis in Ischemic Heart Disease. J. Transl. Med. 15 (1), 87. doi:10.1186/s12967-017-1191-y

Timmers, L., van Keulen, J. K., Hoefer, I. E., Meijs, M. F., van Middelaar, B., den Ouden, K., et al. (2009). Targeted Deletion of Nuclear Factor kappaB P50 Enhances Cardiac Remodeling and Dysfunction Following Myocardial Infarction. Circ. Res. 104 (5), 699-706. doi:10.1161/CIRCRESAHA.108.189746

Titus, A. S., Harikrishnan, V., and Kailasam, S. (2020). Coordinated Regulation of Cell Survival and Cell Cycle Pathways by DDR2-dependent SRF Transcription Factor in Cardiac Fibroblasts. Am. J. Physiol. Heart Circ. Physiol. 318 (6), H1538-H1558. doi:10.1152/ajpheart.00740.2019

Tony, H., Yu, K., and Qiutang, Z. (2015). MicroRNA-208a Silencing Attenuates Doxorubicin Induced Myocyte Apoptosis and Cardiac Dysfunction. Oxid. Med. Cel. Longev. 2015, 597032. doi:10.1155/2015/597032

Travers, J. G., Kamal, F. A., Robbins, J., Yutzey, K. E., and Blaxall, B. C. (2016). Cardiac Fibrosis: The Fibroblast Awakens. Circ. Res. 118 (6), 1021-1040. doi:10. 1161/CIRCRESAHA.115.306565

Trivedi, P. C., Bartlett, J. J., Perez, L. J., Brunt, K. R., Legare, J. F., Hassan, A., et al. (2016). Glucolipotoxicity Diminishes Cardiomyocyte TFEB and Inhibits Lysosomal Autophagy during Obesity and Diabetes. Biochim. Biophys. Acta 1861 (12 Pt A), 1893-1910. doi:10.1016/j.bbalip.2016.09.004

Trivedi, P. C., Bartlett, J. J., Mercer, A., Slade, L., Surette, M., Ballabio, A., et al. (2020). Loss of Function of Transcription Factor EB Remodels Lipid Metabolism and Cell Death Pathways in the Cardiomyocyte. Biochim. Biophys. Acta Mol. Basis Dis. 1866 (10), 165832. doi:10.1016/j.bbadis.2020. 165832

van Berlo, J. H., Elrod, J. W., van den Hoogenhof, M. M., York, A. J., Aronow, B. J., Duncan, S. A., et al. (2010). The Transcription Factor GATA-6 Regulates Pathological Cardiac Hypertrophy. Circ. Res. 107 (8), 1032-1040. doi:10.1161/ CIRCRESAHA.110.220764

van Berlo, J. H., Elrod, J. W., Aronow, B. J., Pu, W. T., and Molkentin, J. D. (2011). Serine 105 Phosphorylation of Transcription Factor GATA4 Is Necessary for Stress-Induced Cardiac Hypertrophy In Vivo. Proc. Natl. Acad. Sci. U. S. A. 108 (30), 12331-12336. doi:10.1073/pnas.1104499108

van Berlo, J. H., Aronow, B. J., and Molkentin, J. D. (2013). Parsing the Roles of the Transcription Factors GATA-4 and GATA-6 in the Adult Cardiac Hypertrophic Response. PLoS One 8 (12), e84591. doi:10.1371/journal.pone. 0084591

Virani, S. S., Alonso, A., Benjamin, E. J., Bittencourt, M. S., Callaway, C. W., Carson, A. P., et al. (2020). Heart Disease and Stroke Statistics-2020 Update: A
Report from the American Heart Association. Circulation 141 (9), e139-e596. doi:10.1161/CIR.0000000000000757

Wang, B., Omar, A., Angelovska, T., Drobic, V., Rattan, S. G., Jones, S. C., et al. (2007). Regulation of Collagen Synthesis by Inhibitory Smad7 in Cardiac Myofibroblasts. Am. J. Physiol. Heart Circ. Physiol. 293 (2), H1282-H1290. doi:10.1152/ajpheart.00910.2006

Wang, P., Liu, J., Li, Y., Wu, S., Luo, J., Yang, H., et al. (2010). Peroxisome Proliferator-Activated Receptor \{delta\} Is an Essential Transcriptional Regulator for Mitochondrial protection and Biogenesis in Adult Heart. Circ. Res. 106 (5), 911-919. doi:10.1161/CIRCRESAHA.109.206185

Wang, J., Liu, X. Y., and Yang, Y. Q. (2011). Novel NKX2-5 Mutations Responsible for Congenital Heart Disease. Genet. Mol. Res. 10 (4), 2905-2915. doi:10.4238/ 2011.November.29.1

Wang, J., Wang, Y., Zhang, W., Zhao, X., Chen, X., Xiao, W., et al. (2016). Phenylephrine Promotes Cardiac Fibroblast Proliferation through CalcineurinNFAT Pathway. Front. Biosci. (Landmark Ed) 21, 502-513. doi:10.2741/4405

Wang, X., Tao, Y., Huang, Y., Zhan, K., Xue, M., Wang, Y., et al. (2017). Catalase Ameliorates Diabetes-Induced Cardiac Injury through Reduced p65/RelAMediated Transcription of BECN1. J. Cel. Mol. Med. 21 (12), 3420-3434. doi: $10.1111 /$ jcmm.13252

Wang, C., Xu, W., Zhang, Y., Zhang, F., and Huang, K. (2018). PARP1 Promote Autophagy in Cardiomyocytes via Modulating FoxO3a Transcription. Cell Death Dis. 9 (11), 1047. doi:10.1038/s41419-018-1108-6

Wei, C., Li, L., and Gupta, S. (2014). NF-kB-mediated miR-30b Regulation in Cardiomyocytes Cell Death by Targeting Bcl-2. Mol. Cel. Biochem. 387 (1-2), 135-141. doi:10.1007/s11010-013-1878-1

Whitcomb, J., Gharibeh, L., and Nemer, M. (2020). From Embryogenesis to Adulthood: Critical Role for GATA Factors in Heart Development and Function. IUBMB Life 72 (1), 53-67. doi:10.1002/iub.2163

Wright, M. B., Bortolini, M., Tadayyon, M., and Bopst, M. (2014). Minireview: Challenges and Opportunities in Development of PPAR Agonists. Mol. Endocrinol. 28 (11), 1756-1768. doi:10.1210/me.2013-1427

Wu, Q. Q., Xiao, Y., Yuan, Y., Ma, Z. G., Liao, H. H., Liu, C., et al. (2017). Mechanisms Contributing to Cardiac Remodelling. Clin. Sci. (Lond) 131 (18), 2319-2345. doi:10.1042/CS20171167

Yamamura, S., Izumiya, Y., Araki, S., Nakamura, T., Kimura, Y., Hanatani, S., et al. (2020). Cardiomyocyte Sirt (Sirtuin) 7 Ameliorates Stress-Induced Cardiac Hypertrophy by Interacting with and Deacetylating GATA4. Hypertension 75 (1), 98-108. doi:10.1161/HYPERTENSIONAHA.119.13357

Yang, X., Li, Q., Lin, X., Ma, Y., Yue, X., Tao, Z., et al. (2012). Mechanism of Fibrotic Cardiomyopathy in Mice Expressing Truncated Rho-Associated Coiled-Coil Protein Kinase 1. FASEB J 26 (5), 2105-2116. doi:10.1096/fj.11201319

Yao, C. X., Xiong, C. J., Wang, W. P., Yang, F., Zhang, S. F., Wang, T. Q., et al. (2012). Transcription Factor GATA-6 Recruits PPARa to Cooperatively Activate Glut4 Gene Expression. J. Mol. Biol. 415 (1), 143-158. doi:10.1016/ j.jmb.2011.11.011

Yuan, J., Chen, H., Ge, D., Xu, Y., Xu, H., Yang, Y., et al. (2017). Mir-21 Promotes Cardiac Fibrosis after Myocardial Infarction via Targeting Smad7. Cell. Physiol. Biochem. 42 (6), 2207-2219. doi:10.1159/000479995

Yuan, F., Qiu, Z. H., Wang, X. H., Sun, Y. M., Wang, J., Li, R. G., et al. (2018). MEF2C Loss-Of-Function Mutation Associated with Familial Dilated Cardiomyopathy. Clin. Chem. Lab. Med. 56 (3), 502-511. doi:10.1515/cclm2017-0461

Yu-Wai-Man, C., Spencer-Dene, B., Lee, R. M. H., Hutchings, K., Lisabeth, E. M., Treisman, R., et al. (2017). Local Delivery of Novel MRTF/SRF Inhibitors Prevents Scar Tissue Formation in a Preclinical Model of Fibrosis. Sci. Rep. 7 (1), 518. doi:10.1038/s41598-017-00212-w

Zeisberg, E. M., Ma, Q., Juraszek, A. L., Moses, K., Schwartz, R. J., Izumo, S., et al. (2005). Morphogenesis of the Right Ventricle Requires Myocardial Expression of Gata4. J. Clin. Invest. 115 (6), 1522-1531. doi:10.1172/JCI23769

Zeng, M., Wei, X., Wu, Z., Li, W., Li, B., Zhen, Y., et al. (2013). NF-kB-mediated Induction of Autophagy in Cardiac Ischemia/reperfusion Injury. Biochem. Biophys. Res. Commun. 436 (2), 180-185. doi:10.1016/j.bbrc.2013.05.070

Zhang, X., Azhar, G., Chai, J., Sheridan, P., Nagano, K., Brown, T., et al. (2001). Cardiomyopathy in Transgenic Mice with Cardiac-specific Overexpression of Serum Response Factor. Am. J. Physiol. Heart Circ. Physiol. 280 (4), H1782-H1792. doi:10.1152/ajpheart.2001.280.4.H1782 
Zhang, C. L., McKinsey, T. A., Chang, S., Antos, C. L., Hill, J. A., and Olson, E. N. (2002). Class II Histone Deacetylases Act as Signal-Responsive Repressors of Cardiac Hypertrophy. Cell 110 (4), 479-488. doi:10.1016/s0092-8674(02)00861-9

Zhang, N., Ye, F., Zhu, W., Hu, D., Xiao, C., Nan, J., et al. (2016). Cardiac Ankyrin Repeat Protein Attenuates Cardiomyocyte Apoptosis by Upregulation of Bcl-2 Expression. Biochim. Biophys. Acta 1863 (12), 3040-3049. doi:10.1016/j. bbamcr.2016.09.024

Zhang, Q., Lenardo, M. J., and Baltimore, D. (2017). 30 Years of NF-кB: A Blossoming of Relevance to Human Pathobiology. Cell 168 (1-2), 37-57. doi:10. 1016/j.cell.2016.12.012

Zhang, X., Lei, F., Wang, X. M., Deng, K. Q., Ji, Y. X., Zhang, Y., et al. (2020). NULP1 Alleviates Cardiac Hypertrophy by Suppressing NFAT3 Transcriptional Activity. J. Am. Heart Assoc. 9 (16), e016419. doi:10.1161/ JAHA.120.016419

Zhou, X. L., Fang, Y. H., Wan, L., Xu, Q. R., Huang, H., Zhu, R. R., et al. (2019). Notch Signaling Inhibits Cardiac Fibroblast to Myofibroblast Transformation by Antagonizing TGF- $\beta 1 /$ Smad3 Signaling. J. Cel. Physiol. 234 (6), 8834-8845. doi: $10.1002 /$ jcp. 27543

Zuo, J., Wu, F., Liu, Y., Xiao, J., Xu, M., Yu, Q., et al. (2015). MicroRNA Transcriptome Profile Analysis in Porcine Muscle and the Effect of miR-143 on the MYH7 Gene and Protein. PLoS One 10 (4), e0124873. doi:10.1371/ journal.pone. 0124873

Conflict of Interest: The authors declare that the research was conducted in the absence of any commercial or financial relationships that could be construed as a potential conflict of interest.

Publisher's Note: All claims expressed in this article are solely those of the authors and do not necessarily represent those of their affiliated organizations, or those of the publisher, the editors and the reviewers. Any product that may be evaluated in this article, or claim that may be made by its manufacturer, is not guaranteed or endorsed by the publisher.

Copyright $\odot 2022$ Hong and Zhang. This is an open-access article distributed under the terms of the Creative Commons Attribution License (CC BY). The use, distribution or reproduction in other forums is permitted, provided the original author(s) and the copyright owner(s) are credited and that the original publication in this journal is cited, in accordance with accepted academic practice. No use, distribution or reproduction is permitted which does not comply with these terms. 


\section{GLOSSARY}

ANP atrial natriuretic peptide

ARC apoptosis repressor with caspase recruitment domain

ATG autophagy-related protein

BNP brain natriuretic peptide

Csx cardiac-specific homeobox

DCM dilated cardiomyopathy

ECM extracellular matrix

EMT epithelial-to-mesenchymal transition

ER endoplasmic reticulum

ERK extracellular regulated protein kinases

FAO fatty acid oxidation

FoxO forkhead box transcription factor $\mathrm{O}$

HDAC histone deacetylase

IאB inhibitor of nuclear factor- $\mathrm{\kappa B}$

IKK inhibitor of nuclear factor- $\kappa \mathrm{B}$ kinase

$\mathbf{I} / \mathbf{R}$ ischemic/reperfusion
MEF2 myocyte enhancer factor 2

$\boldsymbol{\beta}$-MHC $\beta$-isoform of cardiac myosin heavy chain

MAPK mitogen-activated protein kinase

MRTF myocardin-related transcription factor

MI myocardial infarction

NRVM neonatal rats ventricular myocytes

NFAT nuclear factor of activated T-cells

NF- $\mathbf{k B}$ nuclear factor- $\kappa \mathrm{B}$

PPAR peroxisome proliferator activator receptor

ROS reactive oxygen species

SMAD small mothers against decapentaplegic

SRF serum response factor

STAT signal transducer and activator of transcription

TAC transverse aortic constriction

TCF21 transcription factor 21

TGF- $\boldsymbol{\beta}$ transforming growth factor- $\beta$

TFEB transcription factor EB

TRP transient receptor potential 